\title{
DESIGN AND TESTING OF A TOP MASK PROJECTION CERAMIC STEREOLITHOGRAPHY SYSTEM FOR CERAMIC \\ PART MANUFACTURING
}

\author{
A Thesis \\ presented to \\ the Faculty of California Polytechnic State University, \\ San Luis Obispo
}

\author{
In Partial Fulfillment \\ of the Requirements for the Degree \\ Master of Science in Industrial Engineering
}

by

Dylan Robert de Caussin

June 2016 
(C) 2016

Dylan Robert de Caussin

\section{ALL RIGHTS RESERVED}


COMMITTEE MEMBERSHIP

TITLE: $\quad$ Design and Testing of a Top Mask Projection Ceramic Stereolithography System for Ceramic Part Manufacturing

AUTHOR: $\quad$ Dylan Robert de Caussin

DATE SUBMITTED: June 2016

COMMITTEE CHAIR: $\quad$ Xuan Wang, Ph.D.

Assistant Professor of Industrial and Manufacturing Engineering

COMMITTEE MEMBER: Jianbiao John Pan, Ph.D. Professor of Industrial and Manufacturing Engineering

COMMITTEE MEMBER: Tali Freed, Ph.D. Associate Professor of Industrial and Manufacturing Engineering 


\author{
ABSTRACT \\ Design and Testing of a Top Mask Projection Ceramic \\ Stereolithography System for \\ Ceramic Part Manufacturing \\ Dylan Robert de Caussin
}

Ceramic manufacturing is an expensive process with long lead times between the initial design and final manufactured part. This limits the use of ceramic as a viable material unless there is a large project budget or high production volume associated with the part. Ceramic stereolithography is an alternative to producing low cost parts through the mixing of a photo curable resin and ceramic particles. This is an additive manufacturing process in which each layer is built upon the previous to produce a green body that can be sintered for a fully dense ceramic part.

This thesis introduces a new approach to ceramic stereolithography with a top mask projection light source which is much more economical compared to current vector scanning methods. The research goes through the design and development of a stereolithography printer prototype capable of handling ceramics and the testing of different mixtures to provide the best printing results with varying viscosities. The initial testing of this printer has created a starting point for top mask projection as an economical alternative to current ceramic manufacturing techniques.

Keywords: ceramic, stereolithography, alumina, zirconia, resin, mask projection, green body, additive manufacturing, prototype, 3D print 


\section{TABLE OF CONTENTS}

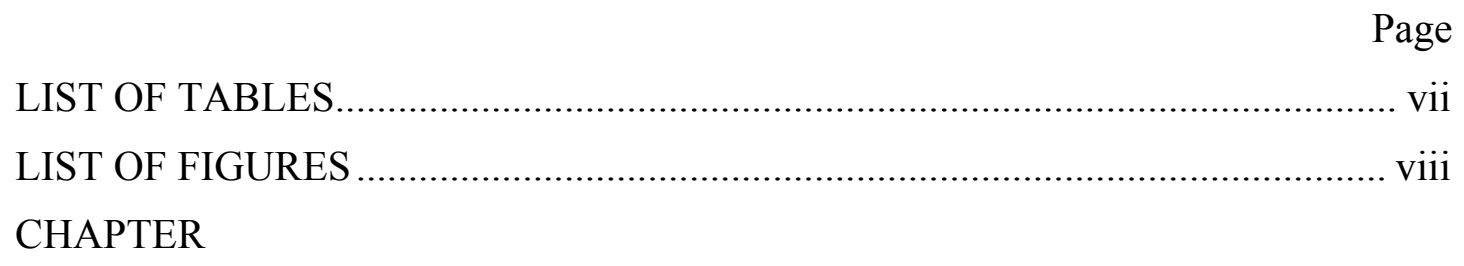

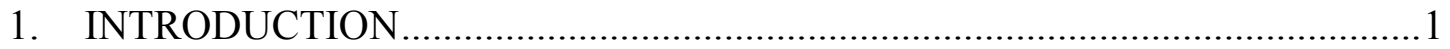

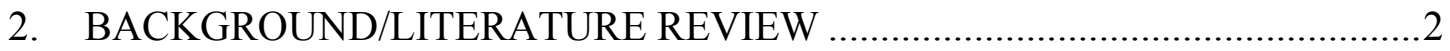

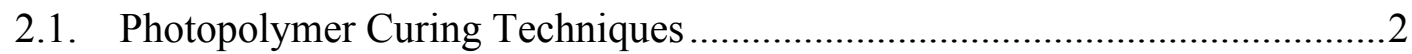

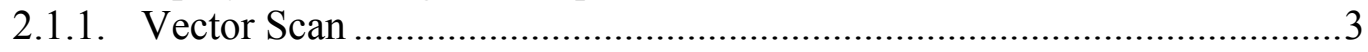

2.1.2. Two Photon ...................................................................................

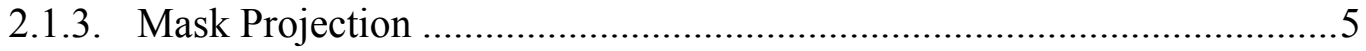

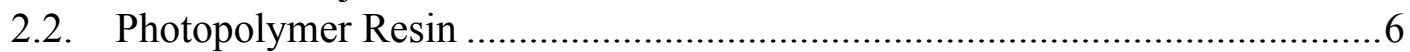

2.2.1. Types of Resin Composition .................................................................

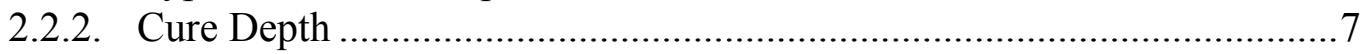

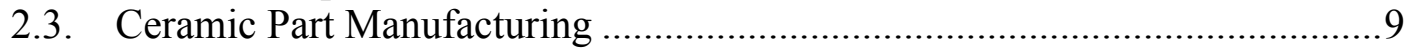

2.3.1. Replication Techniques ...................................................................

2.3.2. Ablating Processes ……………………………................................ 10

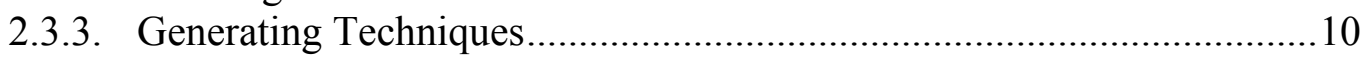

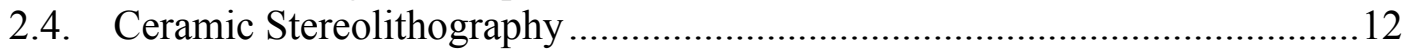

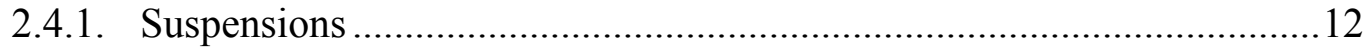

2.4.2. Particle Loading Penetration Depth ....................................................... 16

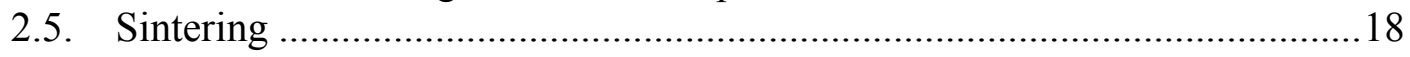

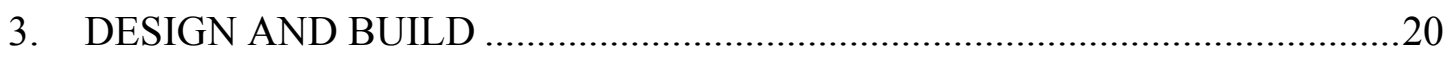

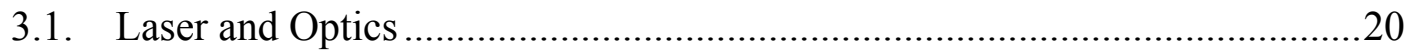

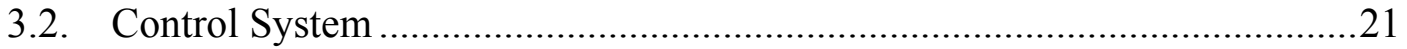

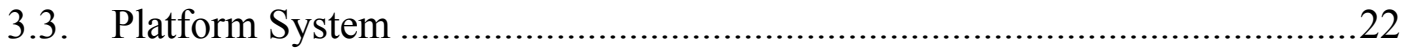

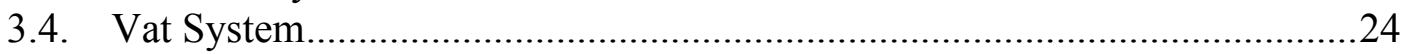

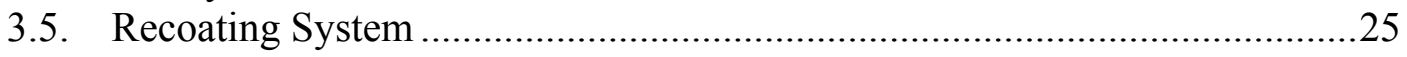

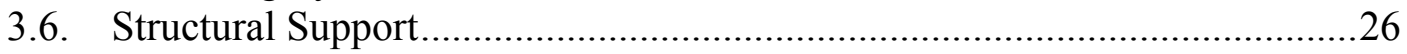

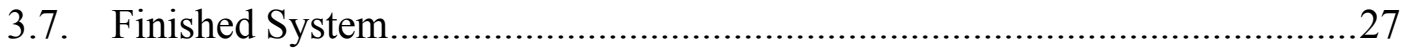

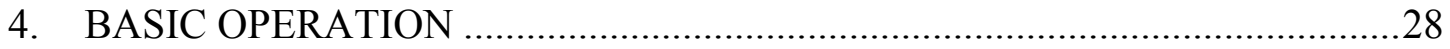

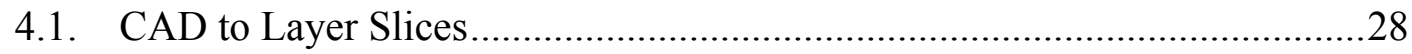

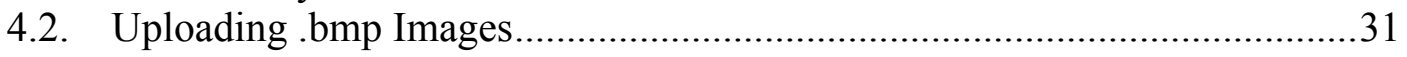

4.3. Create Pattern Sequence ............................................................................

4.4. Prepare Resin .........................................................................................

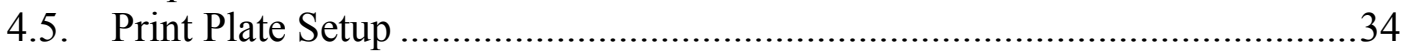

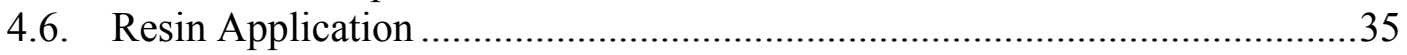

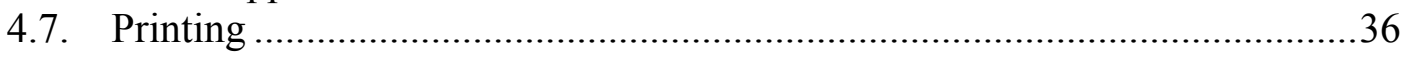

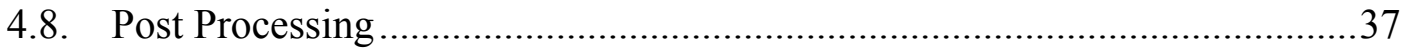

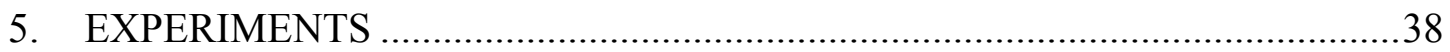

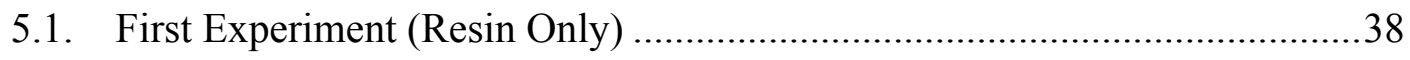

5.1.1. Alphasense Resin Viscosity .................................................................... 
5.1.2. Printing with Alphasense Resin ............................................................40

5.2. Second Experiment (Alumina) ………………..........................................

5.3. Third Experiment (Zirconia) …………………............................................42

5.4. Fourth Experiment (Ceralox and New Resin) ……........................................4

5.4.1. Resin Development ..........................................................................4

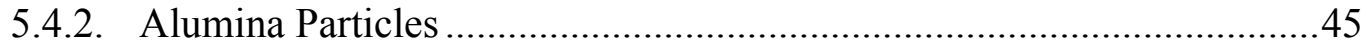

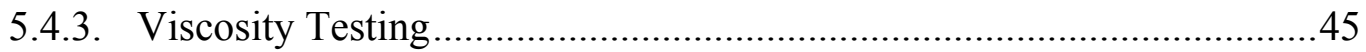

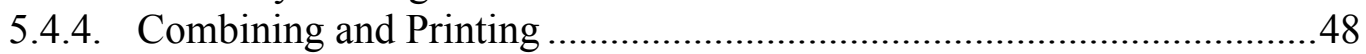

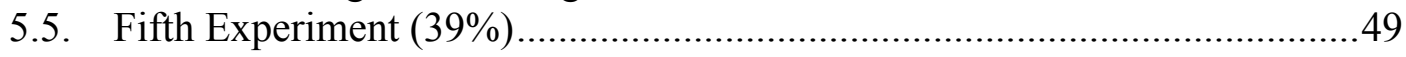

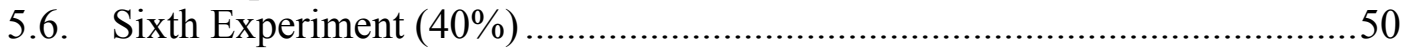

5.7. Sintering ..............................................................................................

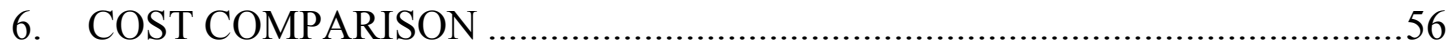

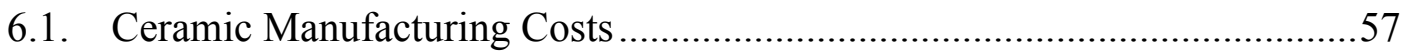

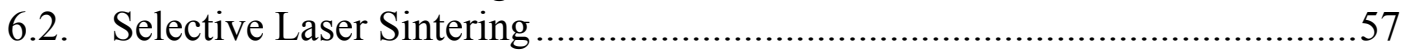

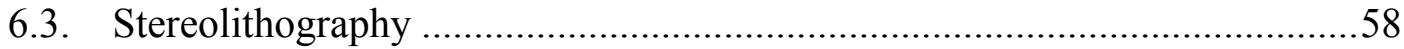

6.4. Top Mask Projection Ceramic Stereolithography ………….........................59

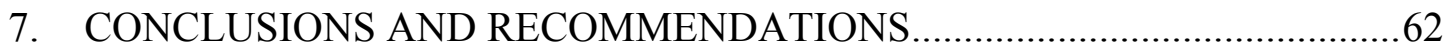

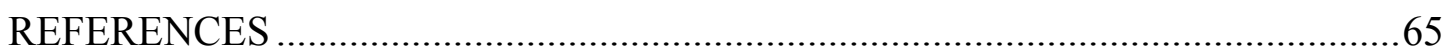




\section{LIST OF TABLES}

Table

Page

Table 1. Reference viscosities for common liquids (Viscosity Values Chart)............15

Table 2. Refractive index of various ceramic materials (Riedel \& I-Wei, 2008)........17

Table 3. Parameters for Freesteel Slicer command window .....................................30

Table 4. Cost breakdown of ceramic stereolithography prototype printer.................59 
Figure

\section{LIST OF FIGURES}

Figure 1. Schematic of vector scan in Stereolithography (Gibson \& Rosen, 2010).....4

Figure 2. Schematic of two photon approach in Stereolithography

(Gibson \& Rosen, 2010).

Figure 3. Schematic of mask projection in Stereolithography

(Gibson \& Rosen, 2010) 6

Figure 4. Viscosity vs \% silica in solution (Griffith \& Halloran, 1996). 15

Figure 5. Viscosity of alumina suspension $(80 \% \mathrm{wt})$ with different diluent percentage versus temperature (Hinczewski, Corbel, \& Chartier, 1998).

Figure 6. Dewaxing process of the green body (Kirihara, 2009).

Figure 7. Wintech Lightcrafter PRO4500 used as lightsource (DLP LightCrafter

4500 Evaluation Module (Rev E)., 2013).

Figure 8. GUI for DLP Lightcrafter to send images and exposure time.

Figure 9. Thorlab manual stage used in the vertical orientation for $\mathrm{z}$ height

(25 mm Translation Stage) 24

Figure 10. Final stereolithography 3D printer with all subcomponents mounted......27

Figure 11. Freesteel Slicer .stl detailed part information display .............................29

Figure 12. Freesteel Slicer example command with printing parameters ..................30

Figure 13. DLP Lightcrafter Control Software firmware changes ............................ 32

Figure 14. DLP Lightcrafter Control Software Pattern Sequence..............................33

Figure 15. Aluminum print plate used for vertical movement. ...............................35

Figure 16. Brookfield viscometer used to test the Alphasense resin.........................39

Figure 17. Alphasense flow curve showing Newtonian fluid behavior. ....................40 
Figure 18. First test parts built from AlphaSense resin showing lifting at base.

Figure 19. Tape casting method with alumina $40 \%$ particle loading.

Figure 20. Zirconia mixture with a $20 \%$ volume particle loading.

Figure 21. Absorbance of photoinitiator at different wavelengths in different

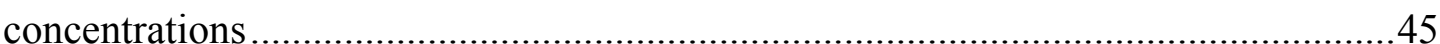

Figure 22. TA Instruments DHR-2 hybrid rheometer used for viscosity testing. ......46

Figure 23. Viscosity test of Spectra resin with alumina in varying volume

percentages.

Figure 24. Ceralox alumina 35\% volume loading printed part.

Figure 25. CP logo printed with 39\% Ceralox alumina. .50

Figure 26. Cal Poly Mustang printed in three different sizes to compare details........51

Figure 27. SunFire 10 furnace used to sinter alumina parts...................................52

Figure 28. Resin burnout initial stages showing carbonization...............................53

Figure 29. Post sintering of the part showing the red hot temperature.......................54

Figure 30. Cooled down part post sintering. ....................................................54

Figure 31. Cost comparison between different ceramic manufacturing processes. ....58 


\section{INTRODUCTION}

Ceramic manufacturing produces parts with desirable traits like high hardness, high temperature strength, and low conductivity. They are used in many different technical fields and have a wide range of applications. This thesis explores the manufacturing process of ceramic stereolithography using mask projection to produce ceramic parts as an alternative to current manufacturing processes. These current processes have high tooling costs and a much longer lead-time to produce a part. The research will investigate the feasibility of using additive manufacturing to lower costs and lower the entry barrier into ceramic manufacturing. It will also use commercially available

products in order to allow this printer to be used without an extensive knowledge of rheological properties and resin structures. As the ceramic stereolithography prototype printer is developed and tested, it will give a better understanding to which variables have the largest impact on the end green-body part produced. The first section is focused on the design and build of a stereolithography printer capable of printing ceramics. Then the testing and experimentation will be done to understand the variables that need to be controlled in the process and how they affect the print quality. Since the design is an iterative process, the end goal is to produce a ceramic stereolithography printer capable of printing comparable parts with the variables understood from a first hand point of view. 


\section{BACKGROUND/LITERATURE REVIEW}

Commercialized additive manufacturing technology has been around since the 1980s and has become more cost effective as technology has advanced. Currently, the most popular and well known process of additive manufacturing is the use of fused deposition modeling (FDM) due to its ease of use, simple technology, and low entry level cost. Stereolithography, however, was one of the first techniques originally developed for additive manufacturing. This process uses ultra-violet radiation from a laser in order expose liquid monomers so that they polymerize (Jacobs, 1996). Under the category of stereolithography, there are three subcategories that describe how the liquid monomer is cured: vector scan, two photon, and mask projection (Gibson \& Rosen, 2010). Another important aspect in stereolithography is the photopolymer composition and how its characteristics affect the build part. Since these are monomer based and yield plastic parts, the material properties are not always sufficient for different engineering applications. In order to change material properties, the photopolymer liquid resin can be combined with a ceramic powder in order to create a ceramic green body. This can then be sintered for a fully ceramic part. Compared to current ceramic manufacturing processes, ceramic stereolithography has many benefits with cost and speed of prototyping. This review of current literature will then give the next steps that need to be explored in the field of stereolithography and ceramic suspensions.

\subsection{Photopolymer Curing Techniques}

When building each layer of a part, there is a build plate below the surface of the resin that each layer is attached to subsequently. When the UV laser touches the 
liquid, it causes cross-linking which hardens the polymers so that the next layer can be built on top. These are three different ways in which photopolymer resins can be exposed to create different layers.

\subsubsection{Vector Scan}

In vector scanning, a laser moves across each point of the part to harden the liquid polymers. The laser is moved using mirrors to cure one entire layer. Then the build platform is dropped down in the $\mathrm{z}$ direction, recoated with a layer of photopolymer, and the next layer is built. A schematic can be seen in Figure 1 depicting the layout. It is similar to fused deposition modeling in that the part is built with a single line of material placed at each point. The main factor in vector scanning is the speed and power at which the laser scans over each part. The two need to be balanced in order to create an accurate part and to have each layer bond to each other. If the laser scans too fast or the power is too low, then the cure depth is too shallow and it won't bond properly to the previous layer. If it is too slow or the power is too high, then it can cause the resin to cure too deep which will reduce the accuracy $(\mathrm{Xu}, \mathrm{Luo}, \mathrm{Ma}, \&$ Yang). A balance is needed between the two and it depends on the particular photopolymer being used and the composition. All of the research papers use this exposure method with different types of laser powers and optics because these are the most prominent on the market. 


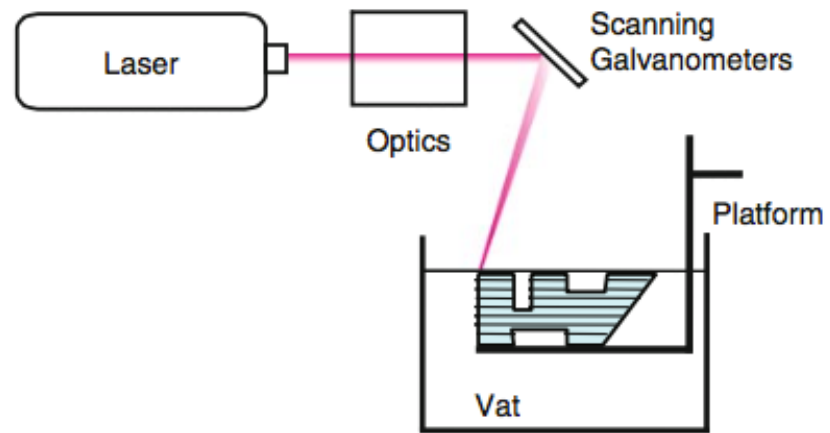

Figure 1. Schematic of vector scan in Stereolithography (Gibson \& Rosen, 2010)

\subsubsection{Two Photon}

Two photon stereolithography is similar to vector scanning except that it uses two intersecting lasers. The schematic can be seen below in Figure 2. When these two lasers intersect, they cause photopolymerization, which hardens the resin at that particular point. This process also scans each layer at a time except there is no need to recoat the build platform. The part is built completely submerged in the vat and the intersection of the two lasers is used to move to a different $\mathrm{z}$ height. This process also has to account for power and scanning speed in order to fully cure the polymer and create a successful part.

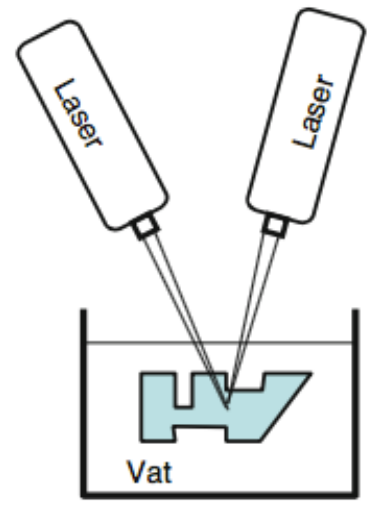

Figure 2. Schematic of two photon approach in Stereolithography (Gibson \& Rosen, 2010) 


\subsubsection{Mask Projection}

In mask projection, each layer of the part is cured at the same time which can be seen in Figure 3. This is accomplished by using a Digital Micromirror Device (DMD) from Texas Instruments. This is a grid of very small mirrors that can either be turned on or off to create an image, similar to how a projector works. As each layer is sent to the control board with the DMD, the mirrors reflect the UV light source onto to the platform and cure that layer. Then the light turns off, the platform moves down, and a new image is projected. In this process, the resin has to flow over the part to recoat each layer, similar to the vector scan. Typically, a blade type device runs over the top of the part to evenly distribute the resin layer thickness. Another way to recoat is to project from the bottom of the part so that the build is upside down and the light source projects under the build platform (Hatzenbichler M. , 2013). This eliminates the need to have a blade for recoat because a vacuum is created when the submerged build plate moves up which then pulls the resin underneath and has a uniform thickness. Both of these mask projection processes are much faster than the vector scan and two photon approach because every layer in the particular cross section is cured at the same time. Rather than having a laser touch every point of the part layer, the mask projection projects the entire layer in a few seconds. The majority of the build time comes from the recoat stage when the polymer has to flow over or under the platform. This technique also doesn't have to worry about cure depth because there is no velocity involved, only the power of the UV light source. Mask projection is typically used for micro scale parts but can be scaled to create larger parts. It also 
has become much more of an open source type of technology since manufacturers are creating their own control boards and the cost has decreased.

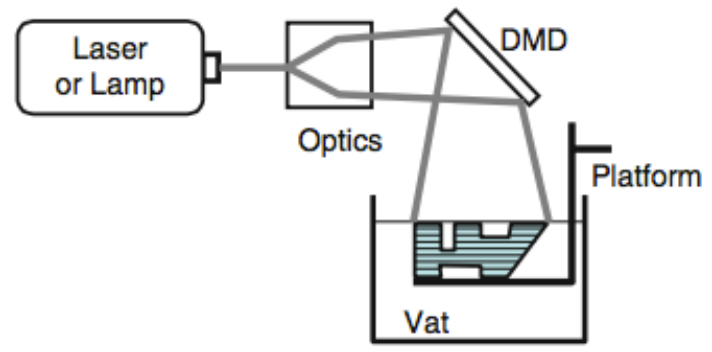

Figure 3. Schematic of mask projection in Stereolithography (Gibson \& Rosen, 2010)

\subsection{Photopolymer Resin}

Photopolymer resins are a mixture of many different elements to create a compound with optimized performance and desired characteristics (Gibson \& Rosen, 2010). One of the essential components in the photopolymer solution is the photoinitiator. This initializes the chemical reaction after it absorbs light and acts as a catalyst. This can be either with UV light, visible light, or any other alternate wavelength source. The most typical wavelength is either UV or some range in the visible light spectrum. When the photoinitiator is exposed, it starts a chemical reaction in which cross linking of polymers is the final outcome. The photopolymer mixture also includes diluents and liquid monomers. The diluents are a reactive substance that helps to lower the viscosity of the solution so it flows easier. The liquid monomers make up a large portion of the structural material of the part being built. Once the photopolymers initiate the chemical reaction, the monomers determine how the cross linking is completed. There are two main types of monomers in the solution: acrylates and epoxies. 


\subsubsection{Types of Resin Composition}

Acrylates are cross-linked by the energy from free radicals (Eschl, Blumenstock, \& Eyerer, 1999). When ultra violet light exposes the mixture, free radicals are released and begin a chain reaction. This chain reaction cross-links the acrylate monomers as long as the exposure is on. Once it is off, the oxygen in the air begins to stop the reaction. Some printers utilize a vacuum space so that the oxygen exposure is limited. Acrylates react very quickly when exposed due to the free radicals but they have large shrinkage rates, which results in curling and warping of printed parts. In order to limit this but utilize the fast reactivity, acrylates are mixed with epoxies.

Epoxies undergo cationic polymerization, which initiates a chain reaction once the UV light reaches a high enough level of exposure (Eschl, Blumenstock, \& Eyerer, 1999). These have minimal shrinkage because the chemical bonds that are formed after are very similar to the beginning chemical structure (Jacobs, 1996). They also take longer to begin cross-linking so the overall curing time is slower. Due to the tradeoffs between the two different monomers, the concentration of epoxies is typically higher than the acrylates. Each distributor of photopolymer resin uses a different ratio in an attempt to maximize performance.

\subsubsection{Cure Depth}

When the UV light hits the surface of the photopolymer, it penetrates a certain depth to begin the chemical reactions. The maximum cure depth $\left(\mathrm{C}_{\mathrm{d}}\right)$ is determined by the ratio between the energy emitted from the light source $(E)$ and the critical energy $\left(\mathrm{E}_{\mathrm{c}}\right)$ 
and can be seen in the equation below.

$$
C_{d}=D_{p} \ln \left(E / E_{c}\right)
$$

The critical energy is the minimum energy needed to begin the chemical reaction in the photopolymer. The energy emitted by the light source is simplified in mask projection because the energy is fairly constant across the surface. The energy emitted is calculated with the irradiance level multiplied by the time of exposure. The irradiance is the radiant power per unit area so the power emitted by the UV light source over the print envelope area. When using a laser curing method, the energy depends on the radius, exposure time, scan speed, and the focus point so it creates many more variables in the system. The mask projection is going to be focused on since that is what is being tested in this thesis. When the light source energy is equal to or greater than the critical energy, the chemical reaction begins. The cure depth also depends on the depth of penetration $\left(\mathrm{D}_{\mathrm{p}}\right)$. This is dependent on the resin and is a characteristic for each which depends on the refractive index. It is quantified as the depth of penetration until a reduction in irradiance of 1/e is reached (Gibson \& Rosen, 2010). In order to increase the cure depth either the light source or the resin can be changed. A more powerful light source will increase the cure depth. A different resin can lower the critical energy needed to start cross linking or can increase the penetration depth. It is important to optimize the cure depth because it determines the layer height of your printer. You need the cure depth to be large enough so that it adheres to the previous layer but not too large that it will begin to over cure below the previous layer. It is an iterative process to get the optimal cure depth and varies between manufacturers so testing is essential to understand resin properties. 


\subsection{Ceramic Part Manufacturing}

Polymer additive manufacturing with stereolithography is a fast and cost effective way to rapid prototype parts. The material properties for these parts are not always suitable for mechanical structural applications and this is where other manufacturing processes come in. Ceramic parts have high hardness and temperature resistance as well as being chemically stable so they can be used in applications where polymers are not acceptable. They also have a high strength to weight ratio as well as low electrical and thermal conductivity. There are three main manufacturing processes in which ceramics are manufactured: replication, ablation, and generation (RitzhauptKleissl, 2009). This thesis will focus on the generation aspect of ceramic part manufacturing but it is important to understand the other processes to see the strengths and weaknesses within each process.

\subsubsection{Replication Techniques}

Replication techniques are the most commonly used methods to create ceramic parts and include ceramic injection molding, die pressing, and slurry casting. These processes all involve molds to shape the parts so there is a high upfront tooling cost but will allow for a higher volume of production. The difference between the replication techniques is how the molds are manufactured or utilized. A powder with binder is typically used in these molds which can lead to limitations in part geometries. This is because the powder needs to be able to fill the entire mold so very fine details are challenging. The part also needs to be able to be removed from the mold so some geometry is not possible to create. After the parts are removed from the molds, they need to be sintered to burn out the binder and increase the part density. 
These replication techniques are all used when volume is needed rather than a prototype or one off part due to the cost and time required to begin production.

\subsubsection{Ablating Processes}

The ablating process takes a fully sintered, hard ceramic part and uses material removal processes in order to shape it to the desired geometry. This involves mechanical machining, laser ablation, and potential EDM processes. The mechanical machining involves using a $\mathrm{CNC}$ or similar machine to cut away from the initial part. Since ceramics are hard materials, the tool wear is very high in this process and can be a large part of the cost. It also limits some of the geometries that can be created based on tool size and typical machining processes. The laser ablation uses a high power laser to etch away at the ceramic and has a high tooling cost. The EDM is possible only if the ceramic is electro conductive. Since most ceramics have a low conductivity, this is typically not an option and is costly. The ablation processes are good when very high tolerances are needed because the shrinkage has already occurred post sintering.

\subsubsection{Generating Techniques}

Generating techniques encompasses all the different types of additive manufacturing processes currently used to print ceramic green bodies. These include selective laser sintering, layered manufacturing, and 3D printing. Generating techniques are very good for rapid prototyping and eliminate the need for any mold design and manufacturing. Selective laser sintering involves a high power laser heating up a powder bed to sinter the ceramic particles. Since ceramics melt at such a high temperature, this requires a lot of power and can lead to local stresses since the 
temperature is very high at once localized point. It is also very difficult to get high precision geometry due to the high temperature needed. Some ways to mitigate this are by using ceramics with lower temperatures to undergo diffusion, which may eliminate some materials as choices. The selective laser sintering machines are very expensive due to the high power laser and mechanics that go into it and may not have the desired surface finish. Layered manufacturing involves cutting or stamping green ceramic sheets layer by layer. Then each one is assembled on top of the next to form a fully green part. After this is can be sintered to achieve the max density of ceramic particles. These machines are also very expensive and have high waste. 3D printing methods encompass many different generating techniques. Binder jetting is a process where binder is applied through a print nozzle and then a powder bed of ceramic is laid on top. Anywhere the binder is applied, the ceramic particles stick together. Material jetting is similar but the binder and ceramic particles are combined and laid down at the same time. This requires a low viscosity material so that it can flow out of the nozzle and still have high detailed parts. The additive manufacturing process that this thesis focuses on is ceramic stereolithography. This involves mixing a photopolymer resin with ceramic particles and using a typical stereolithography exposure method. In order to explore a low cost alternative to ceramic manufacturing, the mask projection technique is used in this paper because it requires less moving parts and the overall system is less expensive than vector scanning. Other researchers have also explored ceramic stereolithography using vector scanning and there is very little in the way of mask projection. In order to optimize this low cost solution, the 
next section details ceramic stereolithography as well as the considerations that should be accounted for.

\subsection{Ceramic Stereolithography}

As discussed earlier, ceramic stereolithography lowers the cost of typical ceramic manufacturing processes and helps to create ceramic parts with better mechanical properties than typical polymers. Ceramic stereolithography uses a UV curable resin to suspend ceramic powder particles so that when the UV light hardens the polymer, it suspends the ceramic particles in a green body (Brady \& Halloran, 1997). This is then put into a furnace to burn out the resin and sinter the ceramic particles to produce

a fully ceramic part. Previous methods for producing ceramic bodies involved compaction in a die and then sintering for a fully dense part. This limited the geometry and the tooling required is very expensive. Ceramic stereolithography allows any geometry to be made that would not be possible with traditional mold methods and at a cheaper cost. In order to build a successful part, there are many factors that need to be controlled in ceramic stereolithography and will be explored in the following sections.

\subsubsection{Suspensions}

The suspension is the material that the ceramic particles are going to be added to. This has a photopolymer to harden the material. When choosing a suspension, the viscosity is a very important aspect and has to be balanced. One aspect of the viscosity is that it should be low so that it can flow and recoat layers easily. If the viscosity is too high, it will be like a gel and a recoating feature will be needed on the stereolithography printer. On the contrary, you want a high viscosity so that when the 
ceramic particles are added, they don't sink to the bottom and fall out of suspension. If the viscosity is close to water, then the particles will conglomerate and sink to the bottom. There is a fine balance between the two that needs to be found experimentally and through testing. Once you have a starting resin with an optimal viscosity, you then have to add the ceramic particles, which increases the viscosity. These are typically added in $40 \%$ to $55 \%$ volume of ceramic particles to the photopolymer (Griffith \& Halloran, 1996). It is an iterative process in order to get the best balance and researchers typically use either aqueous or resin based suspensions. Aqueous suspensions are a mixture of acrylamide and N-N'-methylenebisacrylamide dissolved in deionized water (Wang, Zhou, \& Li, 2010). Since it is a water base, it has a low viscosity and flows very easily, which makes recoating easy. Even though it has a favorable viscosity, it has many other undesirable traits. An example is that the strength after curing is low which makes it delicate. It also doesn't hold the ceramic particles in suspension well because of the low viscosity. The aqueous suspension needs to constantly be agitated to keep the particles suspended. Research from Griffith and Halloran have shown that aqueous acrylamide suspensions have very good fluidity but the depth of cure is not optimal due to the refraction from the powders (Griffith \& Halloran, 1996). In order to improve the cure depth, changes need to be made to reduce the refraction index and add or remove other elements. Once the green body is created, it also has a high water content due to the deionized water base. In order to avoid distortion and cracking in the furnace, the part needs to be dried to remove as much water as possible (Wang et al., 2010). This adds extra time to the post processing of the build. 
In contrast to the aqueous suspension, resin based suspensions create a much stronger green body after curing but also have a higher viscosity (Wang et al., 2010). This type is the common commercial resin used in stereolithography printers. After the ceramic particles are added, there is a desired viscosity range to optimize the performance of the printer. On the lower end, Brady and Halloran have researched that $200 \mathrm{mPa}-\mathrm{s}$ is the lowest viscosity that will ensure the ceramic particles will be stable against sedimentation (Brady \& Halloran, 1997). This is similar to the viscosity of a typical commercial resin. On the upper end, the resin after adding the ceramics needs to be less than $3000 \mathrm{mPa}-\mathrm{s}$ in order to have enough flow to ensure a good recoat (Griffith \& Halloran, 1996). This is similar to glue. Figure 4 shows how the viscosity of resin changes with the addition of silica ceramics. This can be applied to other ceramics as well with minimal changes to the figure shape. As the volume of silica is increased, the viscosity seems to increase exponentially. This is why most suspensions do not get over $55 \%$ volume ceramic. Table 1 shows common liquid viscosities as a reference to compare the ceramic suspensions at different loading percentages. One centipoise $(\mathrm{cP})$ is equal to one millipascal-second (mPa-s) for the conversion ratio. 


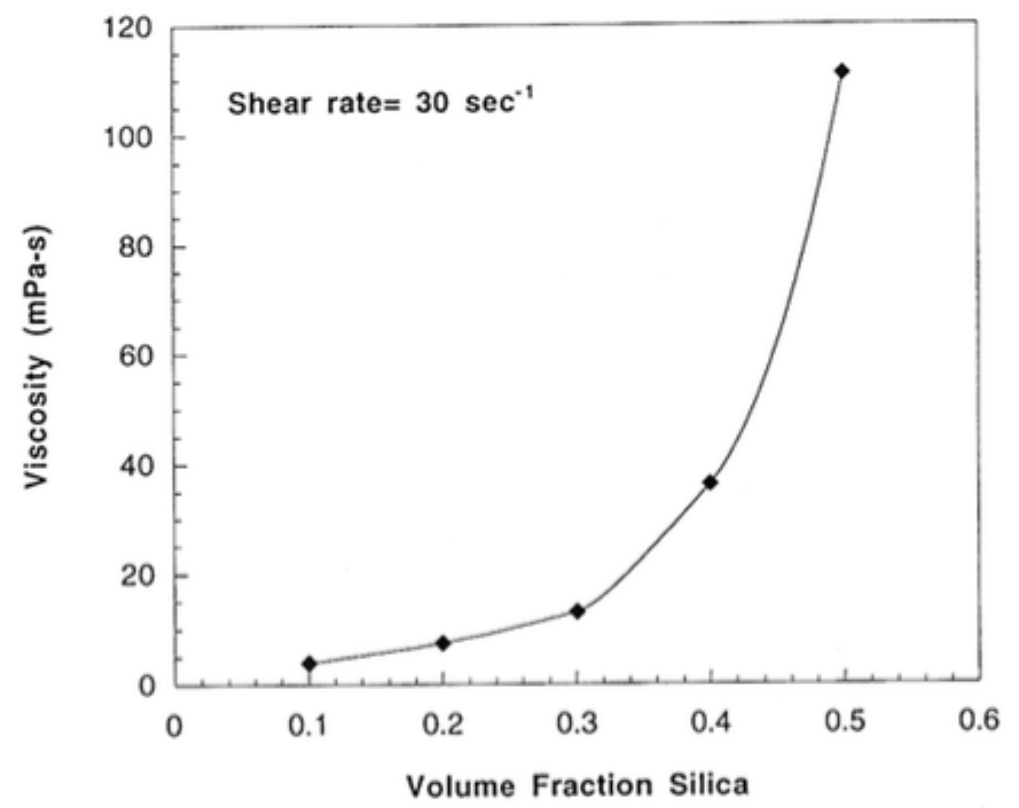

Figure 4. Viscosity vs \% silica in solution (Griffith \& Halloran, 1996)

Table 1. Reference viscosities for common liquids (Viscosity Values Chart)

\begin{tabular}{|c|c|}
\hline Type of Liquid & Viscosity (Centipoise) \\
\hline Water & 1 \\
\hline Milk & 3 \\
\hline Blood & 10 \\
\hline Vegetable Oil & 40 \\
\hline Tomato Juice & 180 \\
\hline Glycerin & 800 \\
\hline Honey & 1500 \\
\hline Glue & 3000 \\
\hline Sour Cream & 15000 \\
\hline
\end{tabular}

In order to get a desirable viscosity, diluents can be added to the resin in order to lower the viscosity or the temperature can be changed. Adding diluents causes the viscosity to decrease, which can be seen in Figure 5. This also shows that as the temperature increases, the viscosity decreases. If a particular resin suspension needs to be lowered significantly, these steps can be taken. 


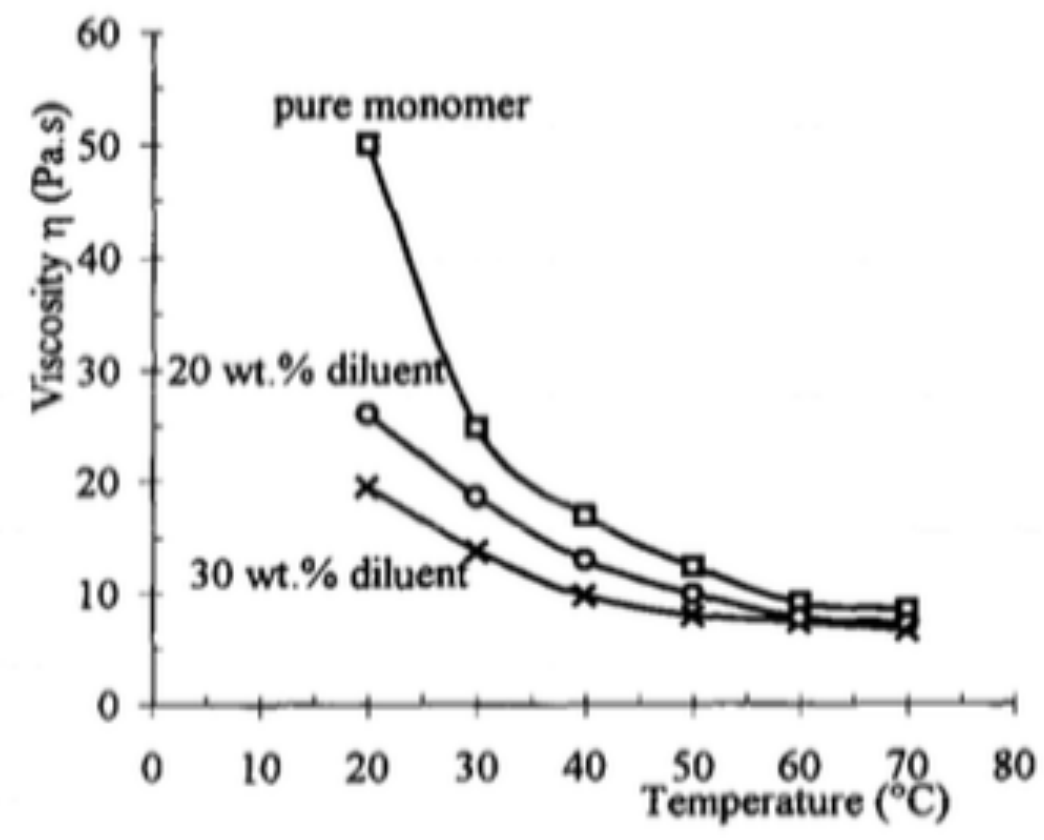

Figure 5. Viscosity of alumina suspension ( $80 \% \mathrm{wt})$ with different diluent percentage versus temperature (Hinczewski, Corbel, \& Chartier, 1998)

\subsubsection{Particle Loading Penetration Depth}

When the ceramic particles are added to the suspension, the cure depth changes as well. The cure depth equation is the same as for the pure resin but the penetration depth $\left(D_{p}\right)$ value changes. Instead of being a constant depending on the resin, it varies depending on the amount of ceramic material. The ceramic particles change the way the light penetrates and can either scatter or absorb to change the refractive index. The equation below shows that penetration depth is proportional to the average particle size (d) and inversely proportional to the ceramic volume fraction $(\phi)$.

$$
\begin{gathered}
C_{d}=D_{p} \ln \left(E / E_{c}\right) \\
D_{p} \propto \frac{d}{\phi} \frac{1}{Q} \\
Q=\beta \Delta n^{2}=\beta\left(n_{\text {ceramic }}-n_{\text {solution }}\right)^{2}
\end{gathered}
$$


The volume fraction is ratio of the volume of ceramic powder to the total volume of the ceramic resin mix. The scattering efficiency (Q) is a function of the refractive index of the two different materials (Griffith \& Halloran, 1996). Even if the ceramic allows light to pass through, there is still some refraction that changes the properties. The difference between the ceramic refractive index $\left(\mathrm{n}_{\text {ceramic }}\right)$ and the resin refractive index ( $\left.\mathrm{n}_{\text {solution }}\right)$ is the largest affect on the scattering efficiency. The closer the two are, the deeper the penetration will be. A reference table for some refractive indexes can be seen below in Table 2 .

Table 2. Refractive index of various ceramic materials (Riedel \& I-Wei, 2008)

\begin{tabular}{|c|c|}
\hline Material & Refractive Index (n) \\
\hline Vacuum & 1 \\
\hline Water & 1.33 \\
\hline Photo Resin & $1.4-1.6$ \\
\hline Alumina & 1.77 \\
\hline Zirconia & 2.05 \\
\hline Silicon Nitride & 2.05 \\
\hline Silicon Carbide & 2.3 \\
\hline
\end{tabular}

The beta term has less affect on the Q. These equations are important because they give us the relationship between the powder and suspension. It is important to have an effective cure depth and this can be accomplished with smaller ceramic particle sizes. When the ceramic is ordered, we can specify a smaller size to get more cure depth. This relationship also shows us that as we increase the volume fraction of ceramic in the solution, it will decrease the cure depth. Too much ceramic will lower the cure depth and also increase the viscosity as talked about earlier. All of these parameters need to be taken into account when selecting the powder to mix into the suspension. 


\subsection{Sintering}

The final step of ceramic stereolithography is taking the green body and sintering it to get the final ceramic part. The sintering process depends a lot on the initial green body that is created. It is important to have as high of a ceramic density as possible because this will limit the shrinkage, which can creep up to $20 \%$ linear shrinkage. To mitigate the effects of shrinkage, it is typical to oversize the green body so that it shrinks to the desired dimension or to post process the sintered part with grinding to dial in the desired dimensions (Ritzhaupt-Kleissl, 2009). When the part is initially put into the furnace, the resin is burnt out which is considered the dewaxing stage (Kirihara, 2009). This is typically done starting at a temperature of 200 degrees Celsius and ramping up to $600^{\circ}$ Celsius in order to avoid any cracking. The ramp rate is dependent on the water content so the wetter the green body, the slower the ramp rate should be to avoid cracking. Some typical numbers for ramp rate based on different papers are between $0.1^{\circ}$ and $2^{\circ}$ Celsius/minute. Figure 6 shows the dewaxing stage and the weight change of the part over the process at the microstructure level. The black at about $400^{\circ} \mathrm{C}$ is the resin carbonizing from the high heat. Then at $600^{\circ} \mathrm{C}$ it turns white, which shows that the resin has all burned out. After this is completed, the part can be sintered at $1580^{\circ} \mathrm{C}$ for 3 hours (Hinczewski et al., 1998). 


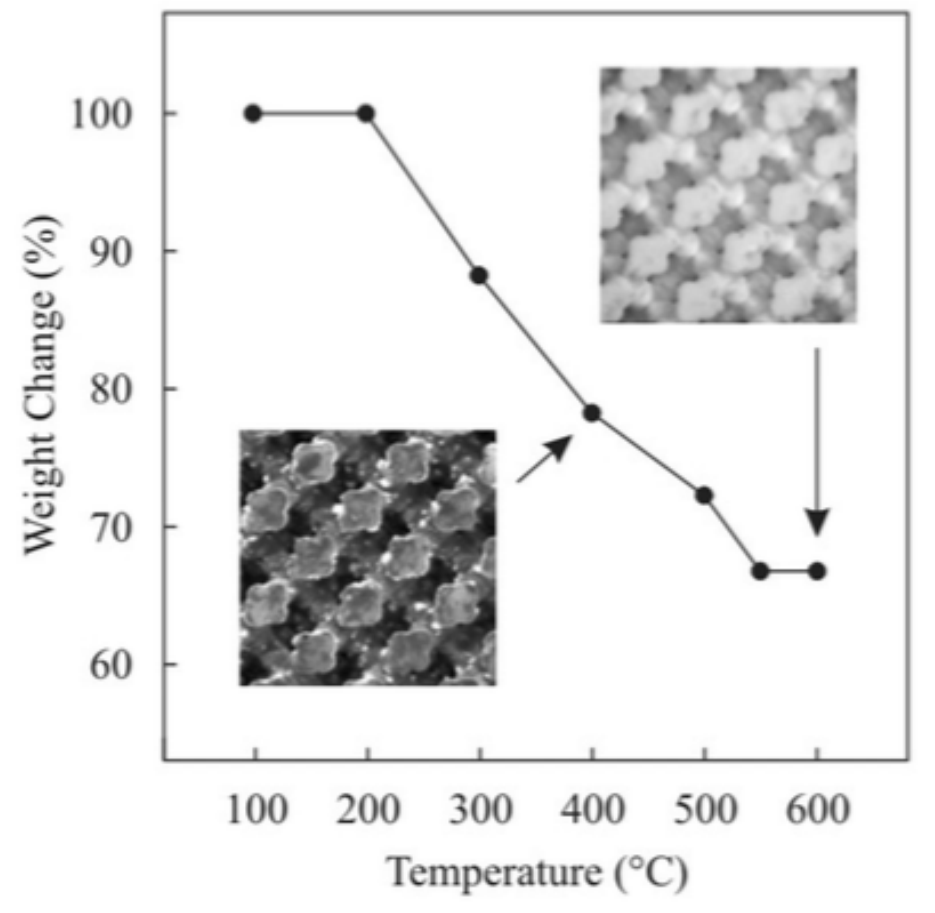

Figure 6. Dewaxing process of the green body (Kirihara, 2009) 


\section{DESIGN AND BUILD}

Since stereolithography printers currently on the market have a high cost associated with them, the first step to exploring this new low cost ceramic manufacturing process is building the printer. In order to build a stereolithography printer, there are multiple components that need to be considered and play a role in the final device working as a system. The first part is the light source and optics, which causes the resin to cure based on the layer section profile. Next is the control system used to connect all the electronics so they expose at the correct time and on the correct layer. Another part of the system is the platform system. This controls the $\mathrm{z}$ height for each layer and moves the print plate to the correct position. The print plate needs to go into a vat system that holds the resin at a constant height and keeps the exposure in focus. The last part of the system is the recoating system, which helps with the resin application between exposures on each layer. All of these were designed to work together and provide a fully functional system. Below is an in depth description of each sub system and how it was designed and used in the stereolithography printer.

\subsection{Laser and Optics}

For the exposure system, the Wintech Lightcrafter PRO4500 was used to create the UV light shown in Figure 7. This off the shelf product is ready to go with a light source and all of the optics in one package. Rather than having to implement each part individually, buying this product saved a lot of time and was a proven concept. Other researchers have used different light sources in order to create similar photopolymer stereolithography printers. The Lightcrafter uses digital micromirror devices (DMD) from Texas Instruments in order to create the exposure profile. It 
redirects the light source, a UV LED, to create the image like a projector would do. The UV LED releases $405 \mathrm{~nm}$ wavelength of light in a field of view of $65.6 \mathrm{~mm} \mathrm{x}$ $41 \mathrm{~mm}$. This is the max printing area per slice that can be produced using this projector. It also has a $92 \mathrm{~mm}$ focal point where the resin surface needs to be. There are also further focal point distances but this was the optimal to provide the printing envelope desired. The other boards on the device allow it to be controlled with a computer. This will be talked more about in the control system.

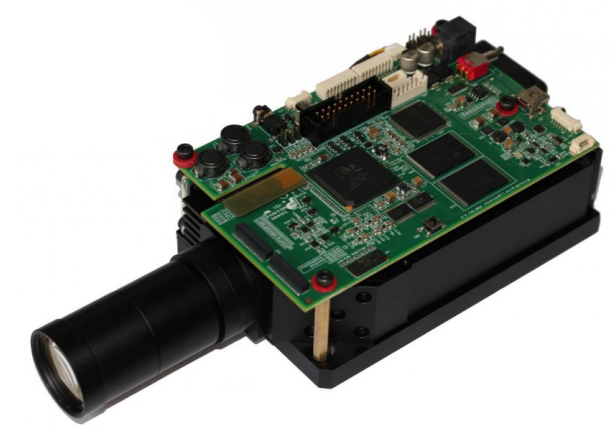

Figure 7. Wintech Lightcrafter PRO4500 used as lightsource (DLP LightCrafter 4500 Evaluation Module (Rev E)., 2013)

\subsection{Control System}

The main part of the control system is communicating with the Lightcrafter what images it needs to display. To accomplish this, Texas Instruments provides a GUI that controls the image upload and exposure time. A screenshot of this can be seen below in Figure 8. The two main ways to control the Lightcrafter are through HDMI or the onboard memory. To control with HDMI, the video mode is selected and the projector streams whatever comes through the HDMI cable. For this project, the onboard memory was used to expose each layer with the pattern sequence mode. Each individual slice is uploaded to the firmware and stored on the external boards. Then when each slice needs to be exposed, it can be selected and added to the pattern 
sequence with a specific exposure time. In order to slice each .stl file, a software called Freesteel Slicer was used which is controlled through the command line interface. It is basic control wise but very powerful in its slicing capabilities once all of the parameters are correctly defined. The software produces a folder of files with each slice in the form of whatever image file is needed. More of the step by step procedure will be talked about later in the basic operations.

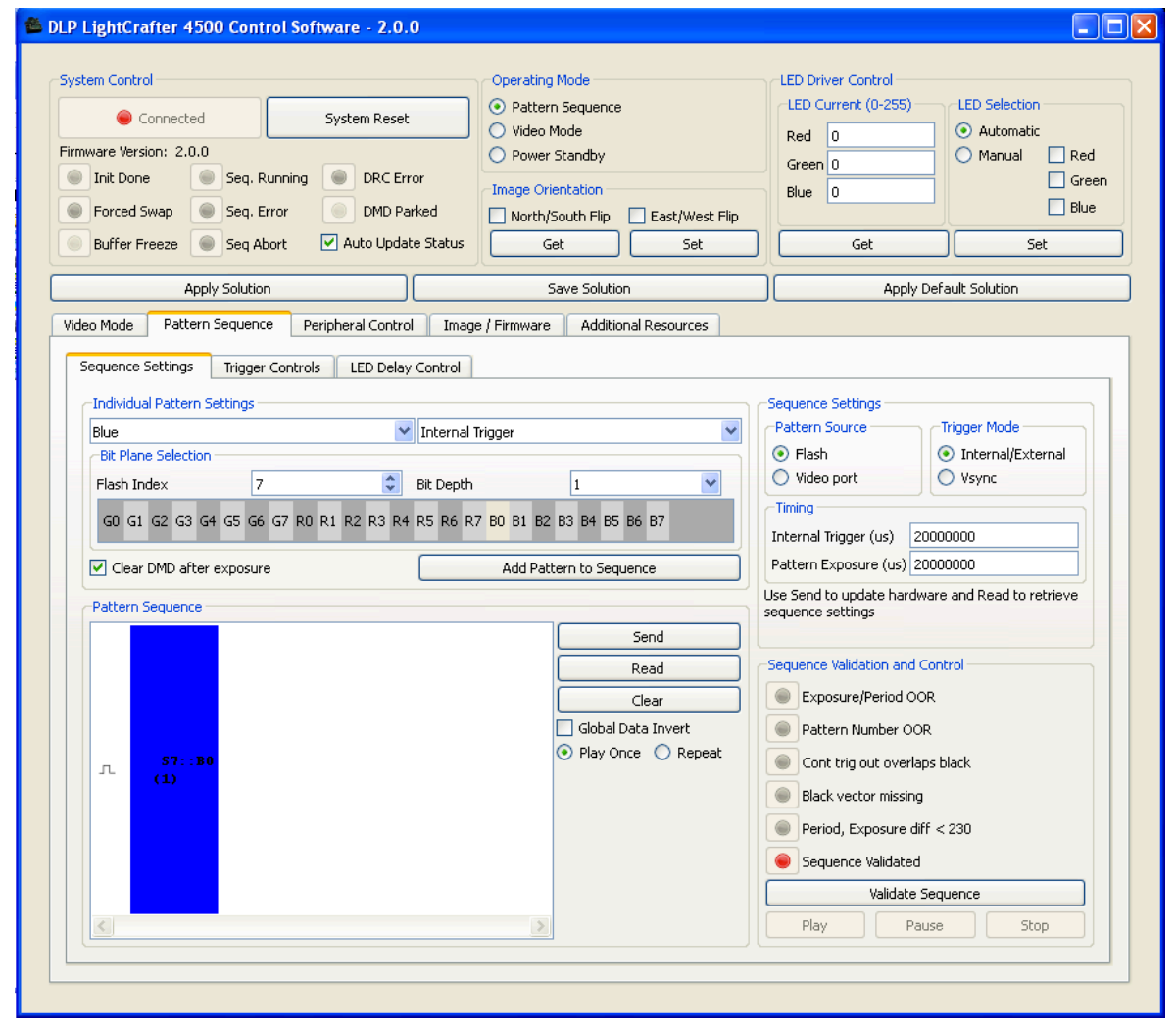

Figure 8. GUI for DLP Lightcrafter to send images and exposure time

\subsection{Platform System}

The platform system moves the printing table in the $\mathrm{z}$ direction between each

exposure. It helps to control the recoating and layer thickness to keep it constant. The recoating is typically achieved by moving the platform a few layers deeper into the resin than the next printing layer. This allows the resin to flow more quickly over the 
top. Then the plate is brought up to the desired layer thickness and allows the excess resin to flow off and resettle. An automatic platform was originally considered to control the step height. This would require a stepper motor with enough accuracy to position the plate at each layer, a system to convert the rotary motion to linear, and a control system to take the computer commands and translate them into motor steps. As each of these parts was specified, it began to increase the cost of the printer and it would almost double the lead-time to build the printer due to the extra components and integration that would be needed. The other issue with this was the communication between the Lightcrafter and the motor. Since the Lightcrafter has its own control software, there was no easy way to accomplish a closed loop with the motor. Either new software needed to be written or an external control board would have to drive the two simultaneously. To solve this, a simple manual stage was used to control the height, which can be seen in Figure 9. This is much less expensive than an automatic system, has more accuracy over the layer height, and is easy to integrate vertically into the system. The actual printing platform that the resin adheres to is an aluminum plate in the shape of an L bracket. This is attached to the vertical stage to move the platform up and down. The material of the print plate is important because the first layer needs good adhesion. After testing and reading about other materials used as print plates, the aluminum was the most successful. A coarser mesh material was experimented with but this did not have good adhesion and made material removal difficult after the part was printed. The end resolution achievable with this set up is 10 microns. 


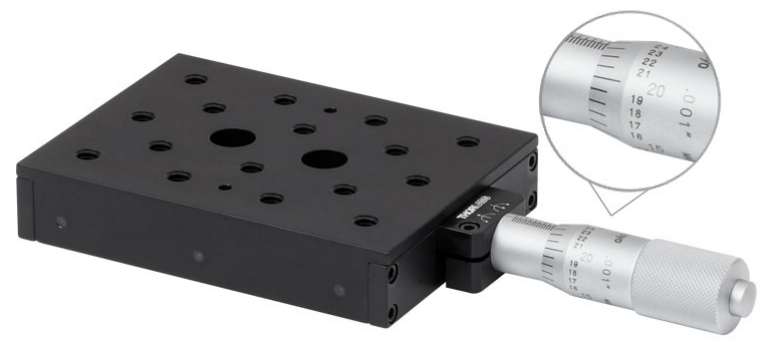

Figure 9. Thorlab manual stage used in the vertical orientation for $z$ height (25 mm Translation Stage)

\subsection{Vat System}

The vat system holds the resin that the printing plate lowers into. The most important aspect of this system is holding the resin at a constant height. This way the UV lightsource stays focused on the top layer. As the plate moves down one layer, the next layer is still in focus because the focal point remains constant. One way to accomplish this is to have a hopper set up to feed resin into the vat if there is any height change. For this project, we used the approach of having a significantly larger vat volume than printing part size. This way the change in height is insignificant because the volume change due to curing shrinkage is low relative to the total volume. This printer has a ceramic pyrex dish marked at the focal point. The ceramic material also allows for easy cleaning between set ups. Another aspect that the vat system potentially needs is a mixer or recirculating system to keep the particles in suspension. The mixer would prevent sedimentation and keep the volume loading constant throughout the entire vat. This is only needed if the viscosity is too low that the resin shows sedimentation during mixing. In order to avoid this, the resin mixture for this printer is going to be targeted to reach the viscosity where there is no sedimentation. The exact numerical viscosity for this varies depending on the weight of the particles and initial resin viscosity. 


\subsection{Recoating System}

The recoating system applies a new layer of resin between each exposure. The approach used for this printer was changed throughout the experiments to try and find the most effective recoating method. First was the simplest method of dipping and raising where between each layer, the platform was lowered past the desired layer height to allow the resin to flow over the existing part. Then once the resin was completely covering, the plate could be raised to the desired height and allow the excess resin to run off to the desired layer height. This approach is time consuming because there is a lot of waiting time for the excess resin to flow off of the part. To expedite this, one approach was blowing on the resin. The air caused to resin to flow off faster but it was also not easily controlled because of the varying strength and difficult repeatability. This resulted in some different layer heights. One solution to this is using a fan that turns on only between exposures to provide a constant source for a specific time. The next approach to altering the dip and raise recoat method was using eccentric motors. Small motors similar to the ones in cell phones were applied to the plate to provide vibration which would cause the resin to flow off quicker as well. This worked well when turned on between layers because the vibration was low enough to provide a constant layer height.

When the ceramic material was applied to the resin, it made the viscosity increase significantly. To print with this new material, we used a tape casting method to apply the resin in layers. The first approach was with a layer template where a known thickness of sheet aluminum had a hole punched in it. This would serve as the layer thickness for each layer applied. Between each exposure, the aluminum sheet was 
placed over the plate and a new layer was applied at the gage thickness. This approach did not initially work because the new ceramic resin had a very low cure depth. This meant that each layer was not adhering to the previous part and nothing was essentially being printed. It was not until a new ceramic resin mixture was created where the cure depth was sufficient enough for this process. Once this was developed, rather than using the template plate to apply each layer, it was directly applied with a squeegee because the template was easily moved and would push the layers off the part. This worked very well but it was difficult to gage the thickness of each layer and therefore they were not uniform. The next step will to be creating a more effective aluminum sheet with a smaller gage and more permanent attachment.

\subsection{Structural Support}

In order to implement these all as a system, a frame was made to hold the parts in place. Poplar was used as the base because this printer doesn't experience any high loads that would require structural materials. Poplar is a hard wood and can hold the tolerances necessary with minimal warping. The only critical features were the mounting holes for the lightcrafter in order to keep it parallel to the ground and print plate. This had paid the most attention to ensure everything was clamped at right angles. The final printer can be seen below in Figure 10 with all the components attached. 


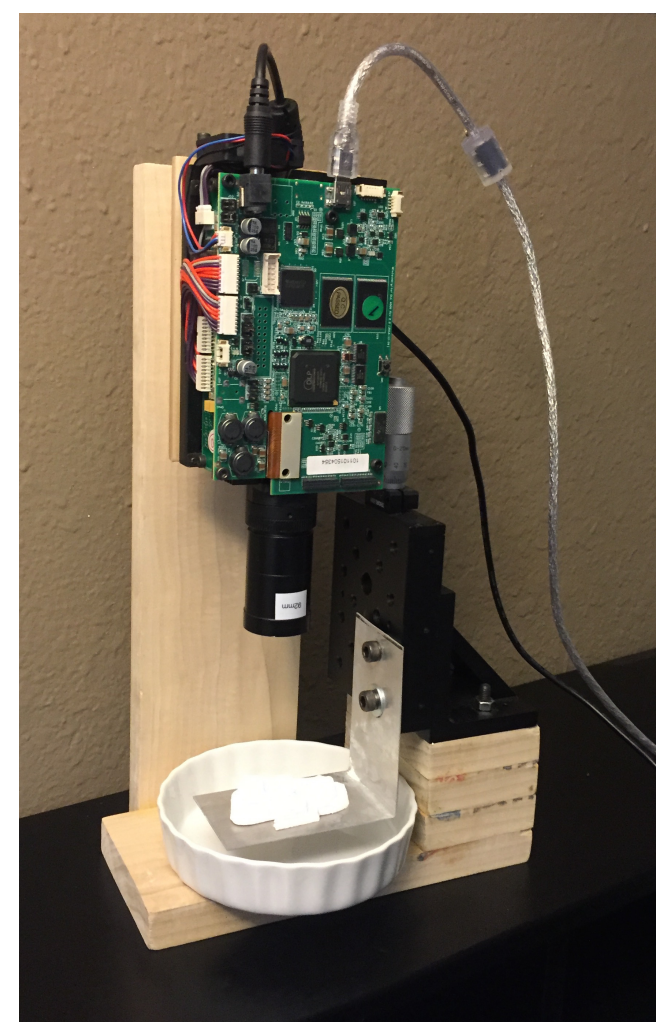

Figure 10. Final stereolithography 3D printer with all subcomponents mounted

\subsection{Finished System}

After going through the initial design ideation and modeling phases, there were some changes that needed to be made in the components that were used. This was because some components did not integrate well into the system and other components had a high price associated with them. This is typical and shows the iterative process in design and more of this is explored when the printer is tested. The changes made help to improve the printer for future use and applications. 


\section{BASIC OPERATION}

For this particular printer, there are certain steps and procedures that need to be completed in order to have a successful part. The following section will go through the operation of this particular stereolithography printer in detail so that it can be used by future research students as well as give an understanding of the entire printing process from 3D model to physical part.

\subsection{CAD to Layer Slices}

The first step in the printing model is to create the model in a CAD program. Once the model is created with the desired geometries, it can be exported as a .stl file. One important modeling aspect that needs to be implemented is the sketch plane of the part due to the slicing freeware used. Freesteel Slicer is a command based application so it has limited configurable settings. When the .stl file is uploaded to the freeware for slicing, it assumes that the direction of slicing is on the front plane. This means that the CAD model part needs to be oriented as if the print plate was the front plane. If the part is oriented in another direction, the slicing freeware will create slices in the wrong direction and the part will not be printed in the desired orientation. This is only necessary for this specific freeware and can be eliminated if another software is available. Once the .stl file is created, the next step is operating the Freesteel Slicer through the command prompt window.

The Freesteel Slicer is a freeware available for download on its website. Once the software is installed on your machine, the command window is opened up to operate it. Before slicing, the correct directory needs to be selected that holds the .stl part file. The next step is doing an analysis on the part to get the relative $\mathrm{z}$ heights that the 
freeware is reading. This is done by typing "slice" followed by the part.stl which can be seen in Figure 11. The output provides all of the dimensions in $\mathrm{mm}$ of the stl that are seen by the slicer with the $\mathrm{z}$ height being the important one for this application. Before moving on to the next step, take note of the zhi and zlo dimensions because these will be an input in the next step.

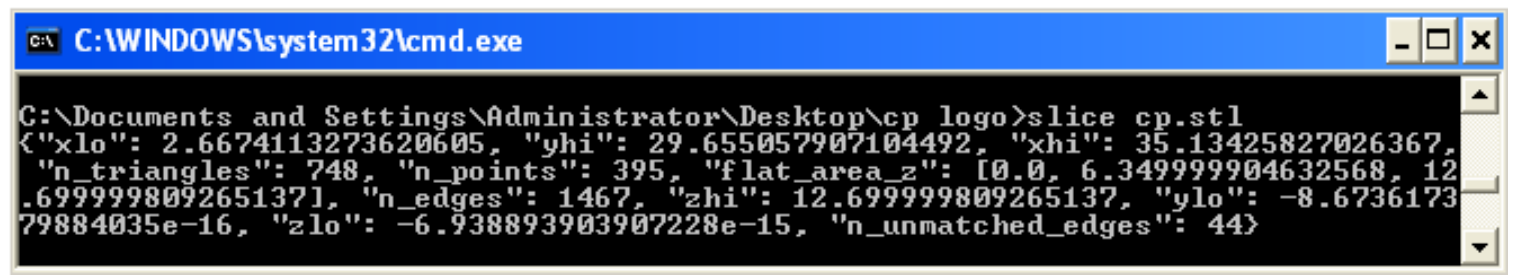

Figure 11. Freesteel Slicer .stl detailed part information display

Now the part can be sliced by inputting "slice" followed by the .stl part as seen in

Figure 12. Everything after this command is the parameters setup for each slice.

Every parameter has a default but after a lot of testing and troubleshooting, there are a few settings that have worked best. There is not a lot of documentation on the different command parameters for this software so Table 3 shows the parameters I have used and what they change. The first setting is the output file designation. This selects the directory that all of the sliced files will be saved in and what the name will be. It also selects the file format which can be in .bmp .png .txt .gif and many more. For this application, the .bmp is used because that is the file that the Lightcrafter control software requires. Next is the cavity and core which designates the colors used to display the slices in the output files. These are set as black and white respectively because the Lightcrafter only exposes at one UV wavelength. The white will be read as the part by the projector and will be the exposed sections. The $\mathrm{z}$ height 
setting uses the previous step of determining the zhi and zlo dimensions so that it sets the upper and lower bounds of the slicing software. The thickness specifies the layer thickness that the software will slice at. Another important parameter is the image resolution that the Lightcrafter takes. It needs to be in HD format so setting the width and height to $912 \times 1140$ is the only way the files will be read. The final parameter is the radius. This freeware can be used for fused deposition modeling as well so this represents the nozzle thickness. For stereolithography slices, the radius is set very small to eliminate any possible tool path marks. A large radius would prevent smaller features from being generated. After all of these parameters are set, the slicing can begin all the files are put into the folder specified.

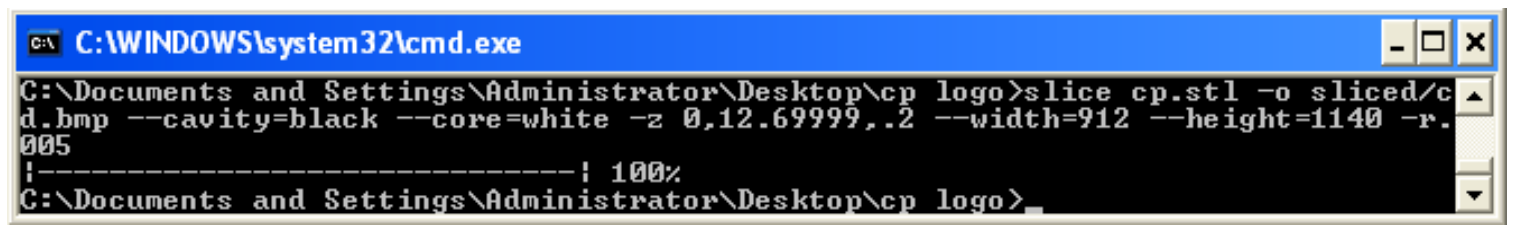

Figure 12. Freesteel Slicer example command with printing parameters

Table 3. Parameters for Freesteel Slicer command window

\begin{tabular}{|l|l|l|}
\hline \multicolumn{1}{|c|}{ Format } & \multicolumn{1}{|c|}{ Optimal parameter } & \multicolumn{1}{c|}{ What it does } \\
\hline -o "output_file_name" & -o folder_name/file_name.bmp & $\begin{array}{l}\text { Sets up the output file name } \\
\text { and the desired file format }\end{array}$ \\
\hline --cavity="color" & --cavity=black & $\begin{array}{l}\text { Makes the background black } \\
\text { in output file }\end{array}$ \\
\hline --core="color" & --core=white & $\begin{array}{l}\text { Makes the part slices white } \\
\text { in the output file }\end{array}$ \\
\hline -z "zlo","zhi", "thickness" & -z zlo,zhi,.2 & $\begin{array}{l}\text { Sets the thickness of each } \\
\text { layer and the z height range }\end{array}$ \\
\hline --width="file width out" & --width=912 & $\begin{array}{l}\text { Sets the output file width for } \\
\text { the image resolution }\end{array}$ \\
\hline --height= "file height out" & --height=1140 & $\begin{array}{l}\text { Sets the output file height for } \\
\text { the image resolution }\end{array}$ \\
\hline -r "toolradius" & -r 0.005 & $\begin{array}{l}\text { Uses a radius to scan/build } \\
\text { each layer }\end{array}$ \\
\hline
\end{tabular}


There is one more step that needs to be completed to upload these generated slices to the DLP Lightcrafter Control Software. After many test prints and exposures using the Lightcrafter, the parts always came out with a skew or stretching factor. When the parts get uploaded, they are stretched by the software even though the images have the correct dimensions. The easiest fix for this is opening the images in a photo software and scaling them in the y direction by 1.25 . There may be another correct way to do this but this was the work around that we came up with that was the easiest and most successful.

\subsection{Uploading .bmp Images}

Now the images are ready to be uploaded with the DLP Lightcrafter Control Software which can be downloaded from Texas Instruments website. The download comes with all the files needed including the firmware files, which the images will be attached to. Under the "Image/Firmware" tab, select "Add Images to Firmware"(see Figure 13). Choose the firmware file from the downloaded folder and then start to add the images by clicking "Add". From this you will be able to select the images from the respective folders and add them to different port numbers on the firmware. This will store each image on the device so they can be used during the exposures. Once the images are uploaded, save the updates to the firmware and go to the "Firmware Update" tab. Connect the Lightcrafter, choose your new firmware file, and upload it to the Lightcrafter. 


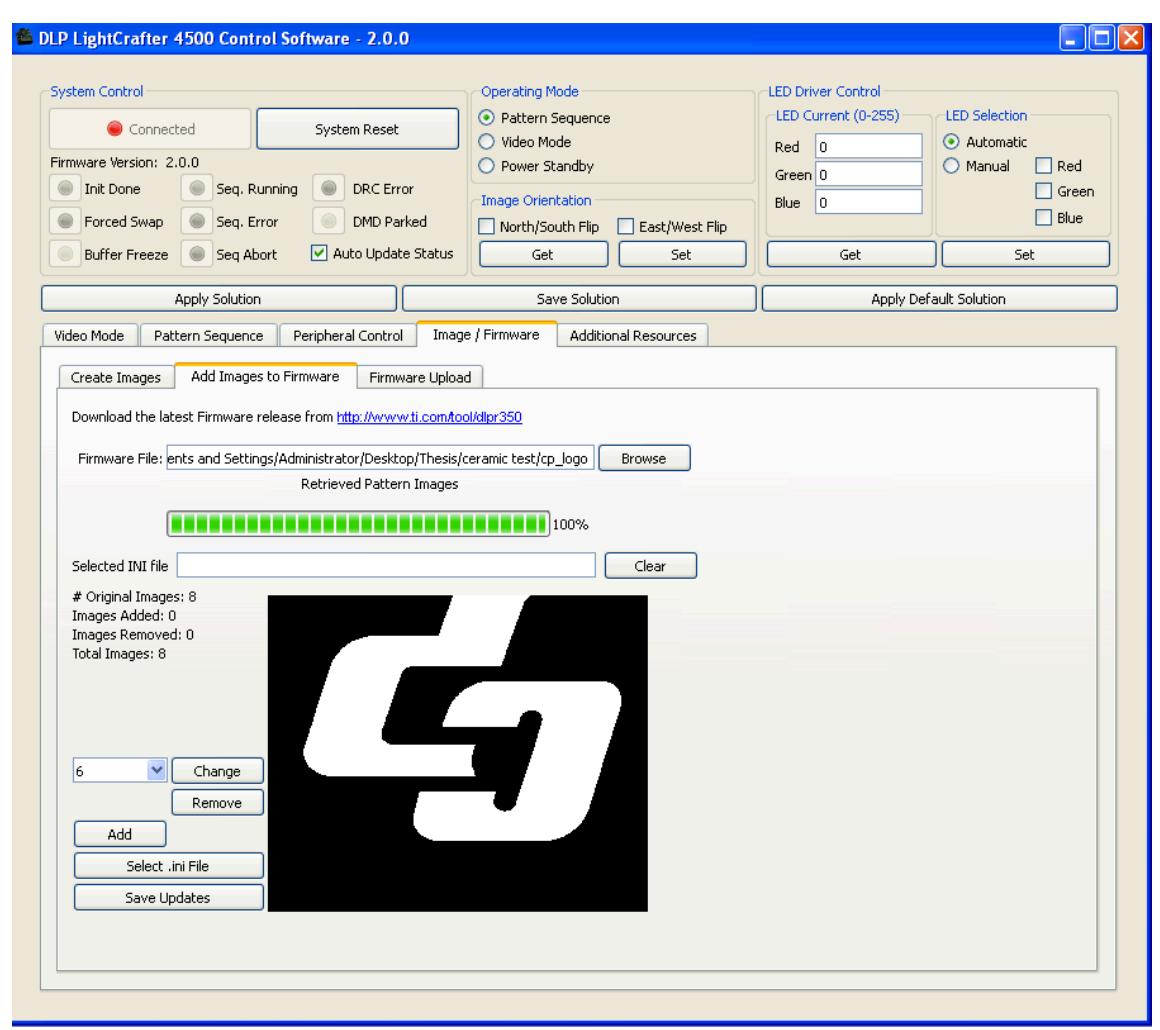

Figure 13. DLP Lightcrafter Control Software firmware changes

\subsection{Create Pattern Sequence}

With the images downloaded onto the Lightcrafter, they can be accessed through the Pattern Sequence tab in the software. First ensure that the "Pattern Sequence" option is selected at the top of the window instead of the video mode. This ensures that the Lightcrafter will read from the firmware instead of the HDMI cable. Now the images can be added by selecting the flash index that matches to the image uploaded previously to the firmware. Each exposure slice has a its own flash index number. Since the Lighcrafter light source is a UV LED, the bit depth and color do not matter because the LED is either on or off. In order to set the exposure time, the far right has an input box to designate the time. There is also an internal trigger box which corresponds to the time in between exposures. This is only needed if a lot of exposures want to be set up in succession. Otherwise each layer slice can be added to 
the sequence between layer changes. To set up the pattern, choose the "Add Pattern to Sequence" button and the blue column will be added with information about the exposure. The next step is either to add more exposures or send it to the Lightcrafter. Adding more slices takes one step out of each exposure process but makes it so each layer has to be applied within that time frame. If each slice is sent individually, there is more flexibility with resin recoating and settling time. Once the pattern sequence is sent, it needs to be validated with the button and then the printing can begin by pushing play.

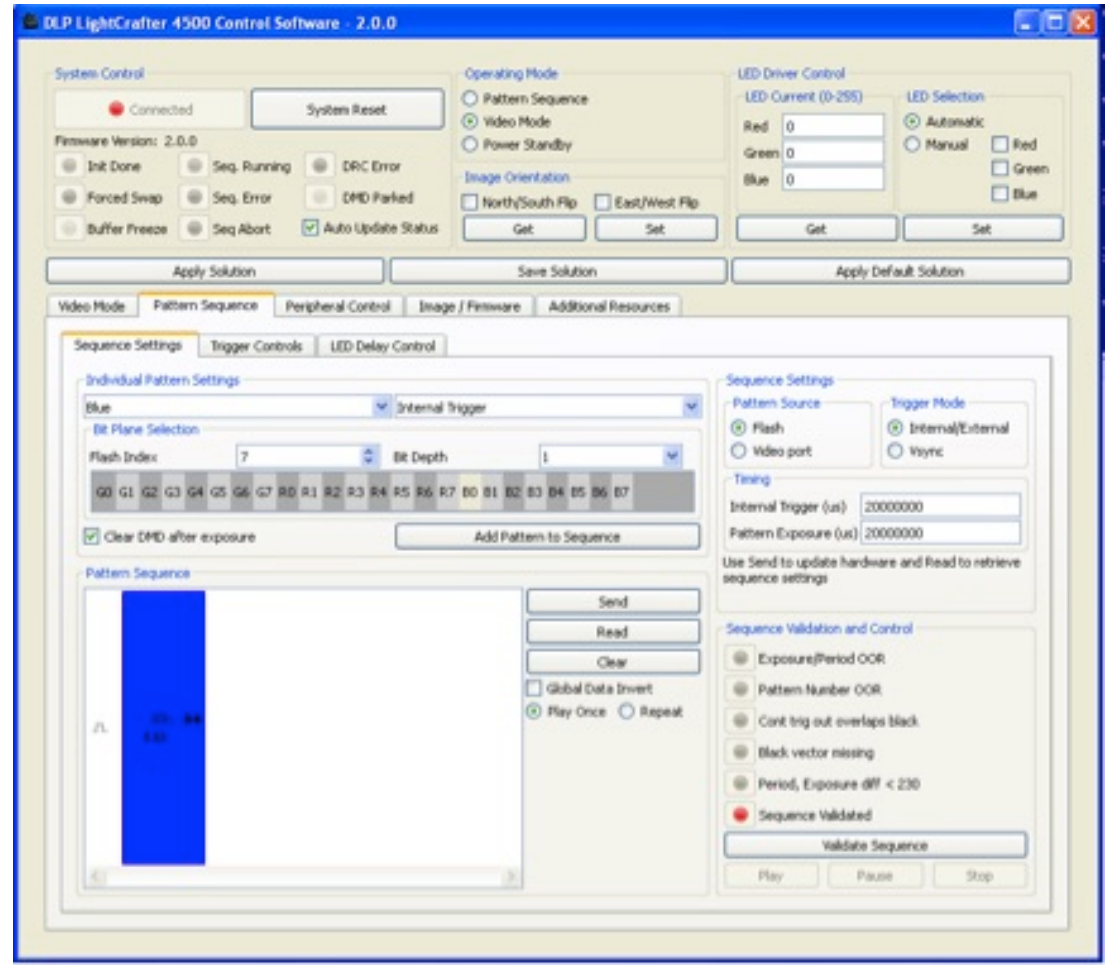

Figure 14. DLP Lighterafter Control Software Pattern Sequence

\subsection{Prepare Resin}

Preparing the material for printing varies significantly depending on the materials being used. The resin may have a higher viscosity or a different cure depth. The viscosity will limit the amount of ceramics that can be added because the end mixture 
will have too high of a viscosity for printing. The cure depth also limits the amount of ceramics because the more particles added lowers the depth that the UV light can penetrate. The best way to go about adding the ceramics to the resin is at a slow controlled rate. First the resin was measured with a syringe to start with the volume in milliliters. Then the ceramic materials were added in incremental amounts. The volume percent was calculated based on the density of the ceramic powder and the mass added to the resin. The initial ceramic particles were added in portions of about 10 percent volume while keeping an eye on the viscosity when mixing. As the viscosity begins to increase significantly, smaller amounts are added to ease the mixing procedure and prevent the end viscosity from being too high. The end viscosity depends on how the printing is going to be accomplished, tape casting or vat method. The final volume percent is the total volume of ceramic particles added divided by the volume of ceramic and resin combined. With this new mixture, the printing can begin for the part.

\subsection{Print Plate Setup}

The first part of the manual operation is adding the print plate to the vertical movement stage. For this printer, the plate is made out of aluminum sheet metal. The resin adheres to an aluminum plate much better than other materials. It is essential to have a strong adhesion to the plate with the first layer because everything builds on top of it. If the layer is not strong enough, it can slide off the plate and the part

dimensions will be incorrect. This is especially true when using a tape casting method because there is a significant amount of shear force on the layers when reapplying resin between exposures. Another important part of the print plate is setting the focal 
point relative to the lens. This particular printer has a focal point of $92 \mathrm{~mm}$ and this can be dialed in with the vertical stage movement. The focal point is important as well because it maximizes the power input into the resin which maximizes the cure depth with ensures good layer adhesion. The last part with the print plate is to ensure that it is parallel to the lens. This ensures that the exposures have a similar exposure power and will create a more accurate final part. Figure 15 shows the final plate made out of sheet metal aluminum to allow for easy fabrication and platform leveling during each exposure.

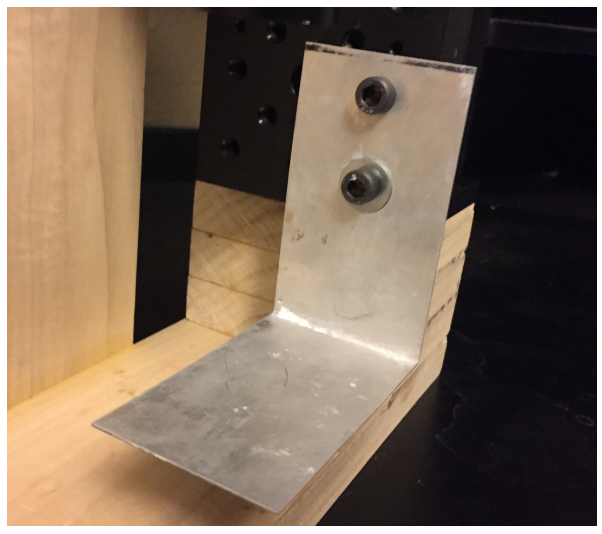

Figure 15. Aluminum print plate used for vertical movement

\subsection{Resin Application}

Next is determining how the resin mixture is going to applied between exposures. The two methods used with this printer are tape casting and a resin vat. The tape casting method is good for higher viscosity materials because the resin doesn't need to flow as easily during the re-coat. It also requires a lot less volume to finish a print which makes it more economical as well. The recoating process is much more involved with this method because after moving down one $\mathrm{z}$ layer, the mixture has to be physically reapplied to the plate. It also has less of a uniform layer because the physical application has human error unless the resin is allowed to settle with long lead times 
between exposures. The resin vat method uses a significant amount of material to print because the top level of resin needs to be at the focal point. This can be limited with a smaller vat or a lower $\mathrm{z}$ travel range. As the layers are exposed, the plate is dipped into the material and then raised to the exposure thickness height. This requires less physical involvement because only the $\mathrm{z}$ height is changed for recoating and the layer thickness is typically more uniform. The one difficulty is ensuring that the resin viscosity is low enough so that the material can flow over each layer and settle down to the correct layer height. The ultimate deciding factor is the viscosity of the printing material.

\subsection{Printing}

Printing the part involves syncing the exposure with the step heights. After the printer is all set up, the first layer of material can be laid down on the print plate. The more uniform the material is placed, the better the final part accuracy will be. To begin the exposure, click the validate sequence button followed by the play button to start the pattern. After the exposure time has finished the pattern created earlier, the $\mathrm{z}$ height can be stepped down one layer thickness and then have the material reapplied. Now the next layer needs to be exposed in the pattern sequence and this pattern follows on until the part is fully created. If the vat method is being used, instead of placing the material on the plate, it is dipped below the surface and then brought up to the exposure height. Then the print plate needs to sit while the excess resin runs off the side for a more uniform layer thickness. T normal exposure procedure can be followed to create the part following this step. 


\subsection{Post Processing}

After the final slice of the print is made, it needs to be removed from the print plate. The easiest way to accomplish this is to first remove the print plate from the $\mathrm{z}$ height. Then deionized water can be used to wash away any of the excess resin that has not been exposed. A small tool is useful to get any resin out of the small thin cracks. After the part is mostly cleaned, it can be removed from the print plate by gently wedging it off with a blade. It is important to go slow because the part is still not fully cured and is fragile. After it is removed, it needs to have a final curing by either leaving it out in the sun or having continuous UV exposure from the lightsource. This allows the resin to fully harden and create a more durable part that can go into the sintering process. 


\section{EXPERIMENTS}

The purpose of these experiments is to show the step by step change to the process based on what was learned from each test. Each test is detailed to give an understanding of what was done and then any shortcomings. As the tests progressed, different aspects of the printer were changed whether it was the material used for printing or the process used on the printer. The experiments show the progressive changes in order to improve the printer and arrive at the final prototype. There are many variables in ceramic stereolithography printing and these experiments show some of the challenges and solutions to improving the final parts produced.

\subsection{First Experiment (Resin Only)}

The first experiment was done to test the printer design and resin composition. A commercial stereolithography resin was used to show that the printer prototype functioned as a stereolithography printer. Once this was confirmed, the next step would be moving on to the ceramic stereolithography. A transparent yellow photo reactive resin from Alphasense was used in this printing session and the first part of the test found the viscosity of the resin.

\subsubsection{Alphasense Resin Viscosity}

Testing the viscosity of the Alphasense viscosity produced a baseline that other resins could be compared to. Using a Brookfield viscometer, the resin was tested with a cylindrical spindle seen in Figure 16. 


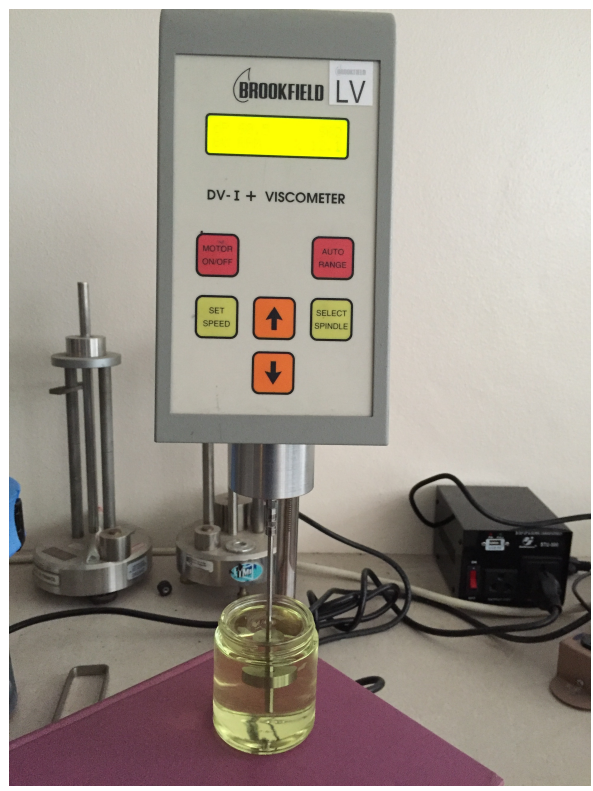

Figure 16. Brookfield viscometer used to test the Alphasense resin

The way this viscometer works is by putting different calibrated spindle sizes into the liquid. As the spindle size increases, the effective area exposed to the liquid increases and more torque is needed to spin in the liquid. The desired viscosity reading is at the point closest to $100 \%$ max torque. In order to get closer to this, either the spindle can be changed or the RPM can be changed. After optimizing this, the viscosity for the Alphasense resin was found to be $37.6 \mathrm{cP}$ at room temperature. Figure 17 shows the behavior of the resin which corresponds to a Newtonian fluid because the viscosity is not changing with shear rate and the shear stress is linearly proportional. This viscosity is low which will be optimal for adding ceramic particles and should keep the ending viscosity lower with higher volume loading. The next step will be to see if adding ceramic particles keeps the mixture Newtonian or how that changes respectively. 


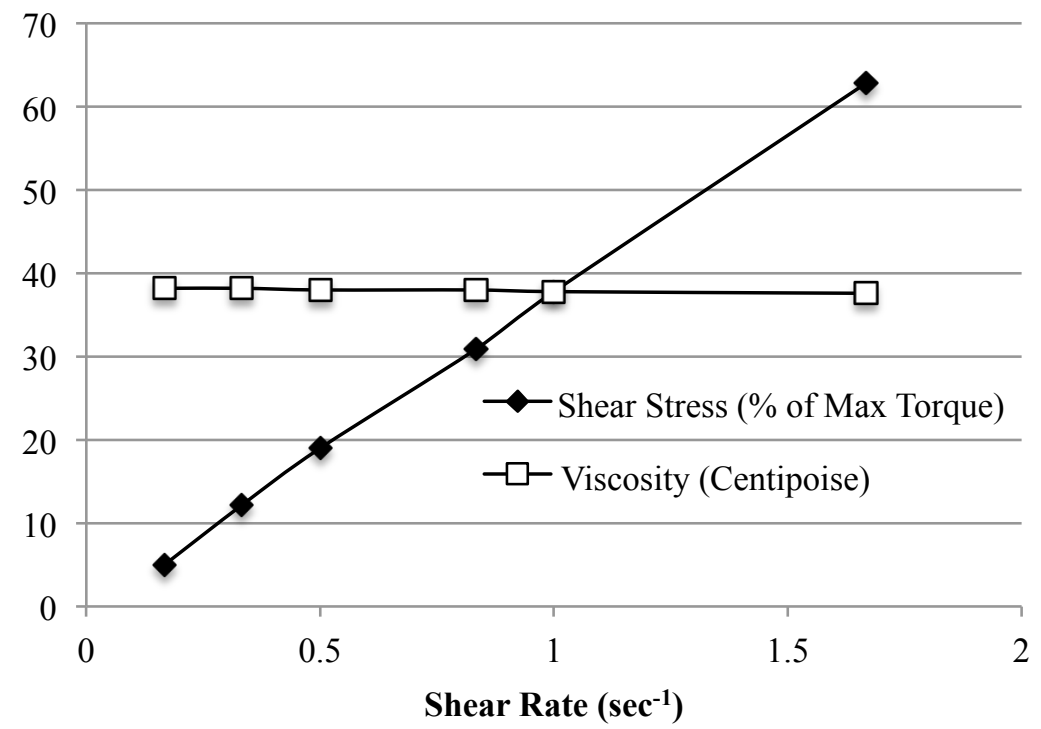

Figure 17. Alphasense flow curve showing Newtonian fluid behavior

\subsubsection{Printing with Alphasense Resin}

Now with the viscosity determined, the procedure for printing the parts was developed. The initial settings for the exposure were 20 seconds of UV light and 40 seconds of rest time in which the plate would step down and the resin would flow to recoat the plate. Rectangle and oval extrusions were used as the test parts with a step size of .1mm per slice. During the exposure, the liquid resin visibly cured when the image was projected. Even though the UV light was on for 20 seconds, the layer was cured in about 5 to 10 seconds. The extra exposure does not affect the final part, it just slows down the print time. For the first 15 exposures $(1.5 \mathrm{~mm})$, we let the resin flow over naturally. However, due to the viscosity and surface tension, each layer did not have a complete recoat. This can bee seen in Figure 18 where the parts have missing structure on the lower half. To fix the surface tension issue, we increased the steps to $.2 \mathrm{~mm}$ and also began to agitate the resin by blowing on it. This ensured a full recoat and the parts slowly began to take on the intended shapes. The blowing also 
allowed for a shorter recoat time than the previous 40 seconds. The final part was 73 steps, which should result in a part $12.7 \mathrm{~mm}$ tall. After measuring, the parts measured 12.6mm, which could be due to some shrinkage when exposed to UV. Overall, this first test was successful in demonstrating the printer's functionality and confirming that the printer can create $3 \mathrm{D}$ parts.

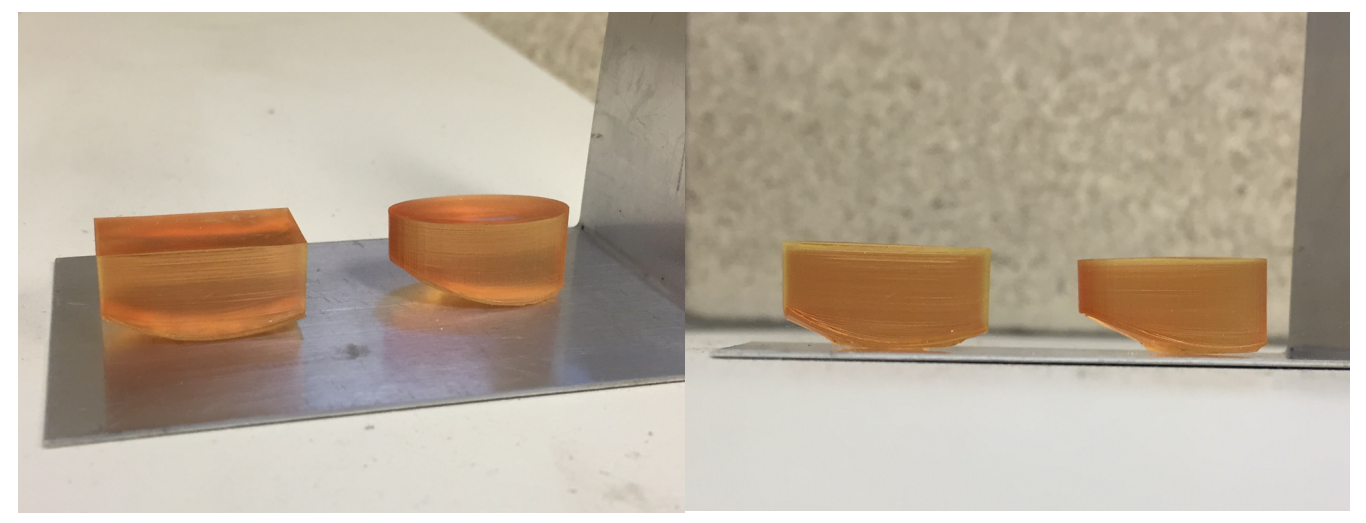

Figure 18. First test parts built from AlphaSense resin showing lifting at base

\subsection{Second Experiment (Alumina)}

For the alumina resin mixture test, the initial particle loading was $50 \%$ by volume. The resin volume used was $10 \mathrm{~mL}$ so $39.5 \mathrm{~g}$ of alumina was mixed in based on a $3.95 \mathrm{~g} / \mathrm{cm}^{\wedge} 3$ density. This mixture resulted in a very powdery end product that was not holding together. It would crumble due to the lack of moisture. To fix this, $5 \mathrm{~mL}$ of resin was added to help with binding and decrease the viscosity. This resulted in $40 \%$ particle loading by volume. The next step was exposing the mixture to see the response. Due to the high viscosity, around $100,000 \mathrm{cP}$, we used a method similar to tape casting to place the mixture on the exposure plate. The mixture was spread evenly over a sheet metal piece with a punched circle in it which can be seen in Figure 19. This would create layers of the same thickness and keep the circular shape. As each layer was exposed, the plate would drop and a new layer would be applied on 
top. After the first exposure of 40 seconds (trying to get the maximum cure depth), the result was a thin film of about 50 microns. This cure depth was not sufficient because it did not allow subsequent layers to bond to the previous. This resulted in the parts being pushed around as each layer was applied which ultimately would not yield a viable part. When exposure time was increased, the cure depth did not increase. This was because the mixture was dark grey, which increased the refractive index and resulted in less UV light absorption. The grey color was due to the alumina grade. Rather than using a pure alumina, which is usually white, our powder was likely intended for polishing purposes. To fix this, we will look for a white alumina with smaller particle sizes to increase the cure depth.

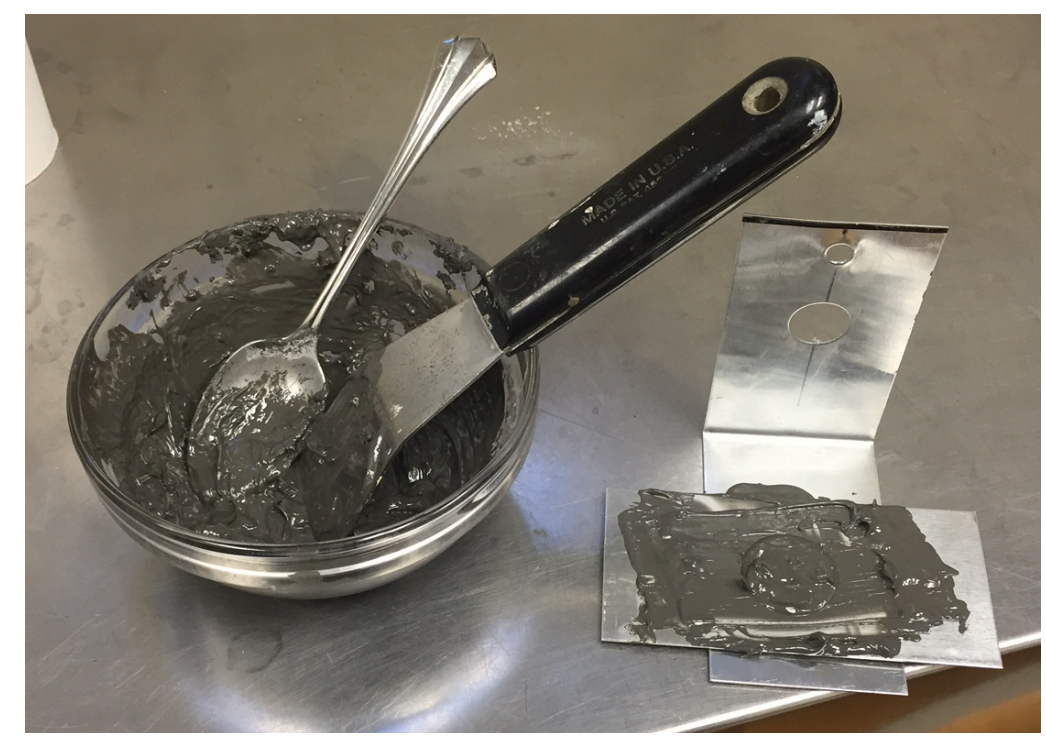

Figure 19. Tape casting method with alumina $40 \%$ particle loading

\subsection{Third Experiment (Zirconia)}

For the zirconia mixture, a lower volume percentage of $20 \%$ particle loading was used to ensure the right viscosity was achieved. If we wanted a higher viscosity, we would then be able to add more zirconia. Zirconia has a smaller particle size than the alumina used earlier which should result in a deeper cure depth. Zirconia also has a 
higher density of $5.68 \mathrm{~g} / \mathrm{cm}^{\wedge} 3$. Using $10 \mathrm{~mL}$ of resin to start again, $14.2 \mathrm{~g}$ of zirconia gave a $20 \%$ volume loading. Figure 20 shows the mixture at this point. The white color will allow for larger cure depth as opposed to the grey alumina mixture. The viscosity of the zirconia $20 \%$ was around $25,000 \mathrm{cP}$ which is still too high for traditional stereolithography printing. To continue with the tape casting method, we increased the particle loading to $26 \%$ which resulted in a similar viscosity to the alumina. Adding an extra $6 \%$ particle loading just about quadrupled the viscosity. When exposing this to the UV light source, we experienced the cured part not adhering to the base plate. This is because the thickness of the tape cast plate is $1.29 \mathrm{~mm}$ and the cure depth is not that deep. To fix this, we simply smeared the mixture on the plate not worrying about the lack of thickness uniformity. When this was done, the layer had a very strong adhesion to the aluminum plate which is what is needed to begin a successful part. If the first layer does not get fixed, then all subsequent layers will not have high accuracy. One way to fix the tape casting is to get a thinner aluminum sheet. This would allow for the thickness to correspond to the cure depth. The cure depth of this zirconia mixture was much higher than the alumina at about $.25 \mathrm{~mm}$ and each subsequent layer was successfully bonding with the previous. One note to consider is that the particle loading was much lower at $26 \%$, which would not be sufficient to provide a fully ceramic part after sintering. The final part also was rough like a 24 grit sandpaper which could be due to the uneven layer addition or the ceramic particles. The next steps are to increase the particle loading but continue to keep the viscosity low. 


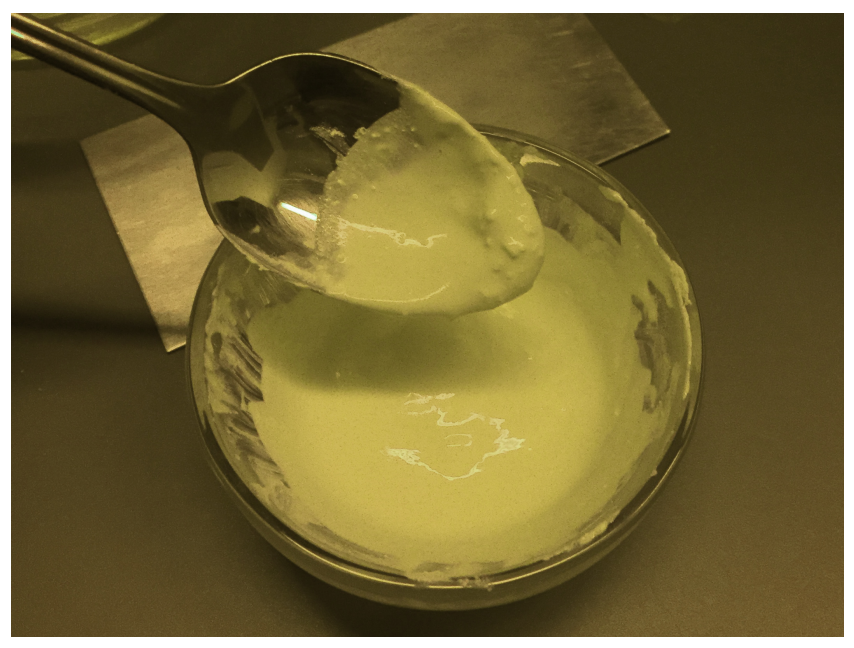

Figure 20. Zirconia mixture with a $20 \%$ volume particle loading

\subsection{Fourth Experiment (Ceralox and New Resin)}

Taking what was learned from the previous two experiments, the resin and ceramic material were changed. Instead of using an off the shelf resin used in stereolithography printers, a custom resin was made for this purpose which was discussed with the resin manufacturer. A new alumina was also found with smaller particle sizes and some dopants in the powder.

\subsubsection{Resin Development}

After talking to the engineers at Spectra Group Ltd. (resin manufacturer), a custom resin formula was made in order to have the desired properties. The resin called IJ111 is an acrylic monomer base with a viscosity of 85 centipoise at 25 Celsius. It has no photo inhibitors added in order to maximize the cure depth. The goal is to have the ceramic particles act as the photo inhibitors and determine the final cure depth. When this resin is exposed to UV light by itself, it can reach a cure depth over one inch depending on the light source power. In order to effectively print with this, typically the light needs to be modulated to control the depth but the ceramic particles will play 
that role in this application. Another feature of this resin is the percentage of photoinitiator present in the mixture. Since the light source emits $405 \mathrm{~nm}$ wavelength UV light, the percentage of $\mathrm{H}-\mathrm{Nu}$ photoinitiator was optimized to maximize the absorbance of the resin. Figure 21 shows the two ends of the spectrum for the concentration of photoinitiator and this resin falls at about $7 \%$.

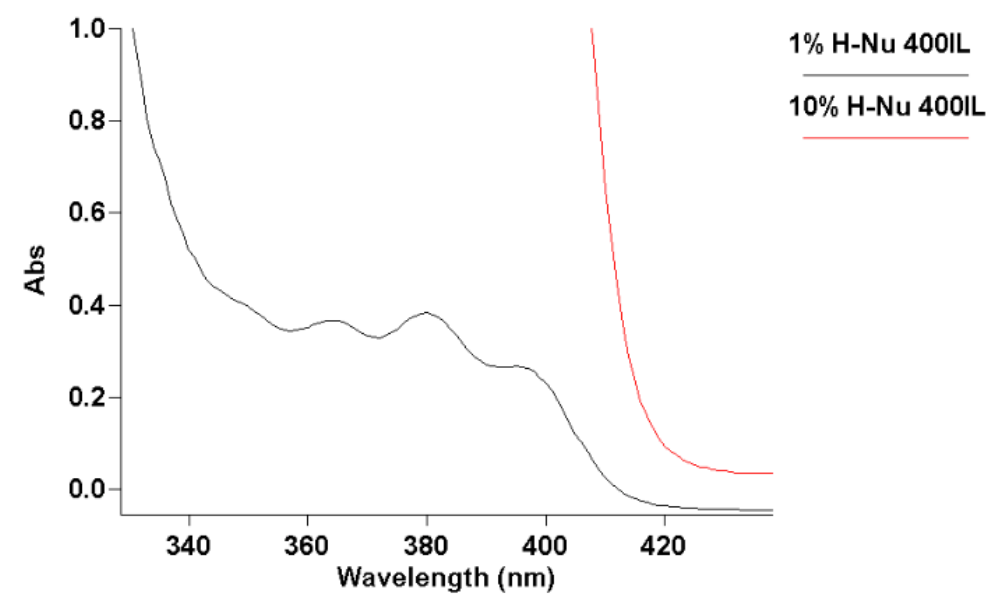

Figure 21. Absorbance of photoinitiator at different wavelengths in different concentrations

\subsubsection{Alumina Particles}

For the alumina powder, Ceralox APA-0.5 was used from Sasol, which has a .5 micron average particle size. The engineer from Sasol suggested doping the alumina with magnesia at $500 \mathrm{ppm}$ to help minimize agglomeration and maximize dispersion. The small particle size and magnesia were chosen to help keep the viscosity lower once it is added to the resin.

\subsubsection{Viscosity Testing}

Testing the viscosity of this resin ceramic mixture was important because of the information it provides about the printing material. As discussed earlier, in order to have a successful stereolithography print with vat recoating, the viscosity needs to be 
below $3000 \mathrm{cP}$ to flow properly and above $200 \mathrm{cP}$ to limit sedimentation. For this test, the DHR-2 rheometer from TA Instruments was used which can be seen below in Figure 22. The sample is placed between a plate and a cone with 55 micron spacing between the two. The cone has a two-degree taper and is attached to the spindle measuring the torque based on shear rate.

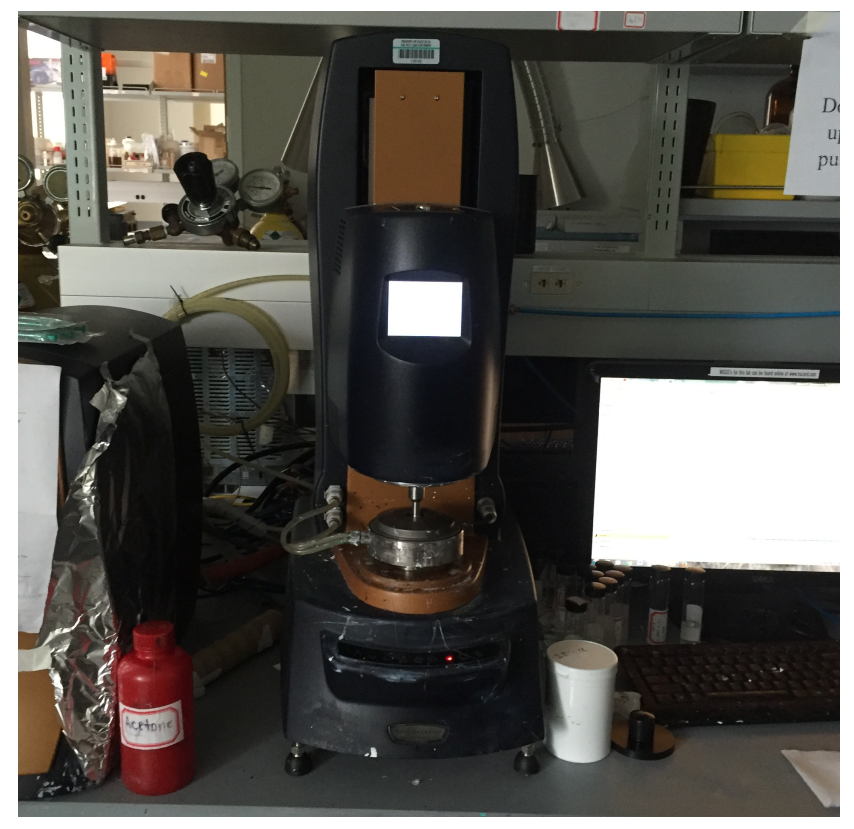

Figure 22. TA Instruments DHR-2 hybrid rheometer used for viscosity testing

The test involved two steps with the first being a flow peak hold and the second a flow sweep. The flow peak hold is an initial step to prep the sample being measured and ensures that the sample is homogenous between the cone and plate. This was done for 60 seconds at $100 \mathrm{sec}^{-1}$ speed. All testing was done at 25 degrees Celsius. The flow sweep tests the viscosity at different shear rates taking the average of the measurements over 60 seconds for each data point. The range of shear rates varied between $.01 \mathrm{sec}^{-1}$ and $10 \mathrm{sec}^{-1}$. This lower shear rate range was used because we wanted to measure the sample around the shear rate it would experience during printing. The first test was done with the resin alone with the next four tests varying 
between $10 \%$ and $35 \%$ volume loading of alumina which can be seen below in Figure 23.

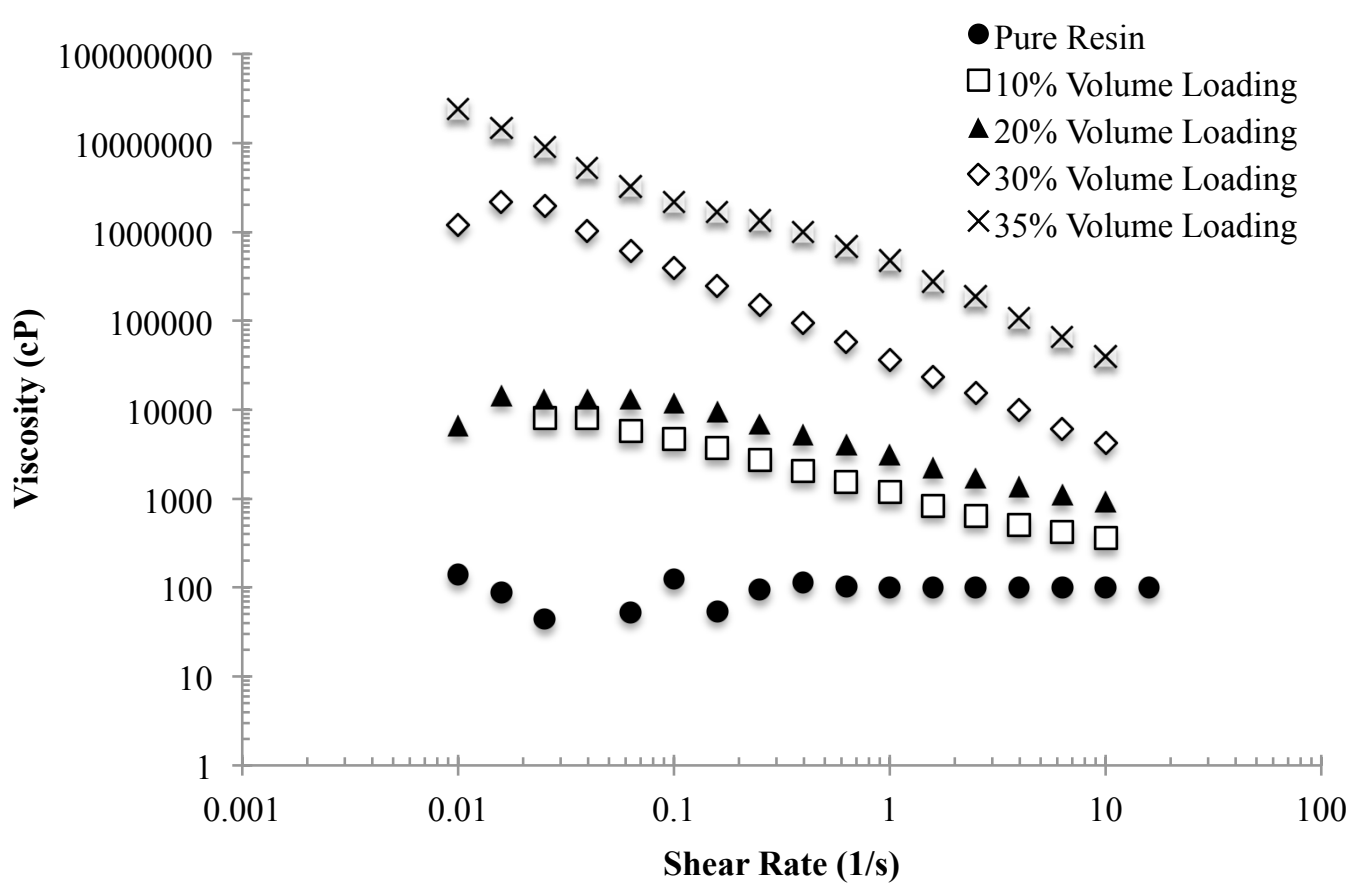

Figure 23. Viscosity test of Spectra resin with alumina in varying volume percentages

The pure resin exhibits Newtonian fluid behavior because the viscosity remains relatively constant with a changing shear rate. It shows a viscosity around 100cP which is close to the manufacturers specification of $85 \mathrm{cP}$. All of the samples with alumina however seem to show a shear thinning behavior which means that as the shear rate is increased, the viscosity decreases. This is probably due to the alumina being a suspension in the resin rather than a complete uniform dispersion. One important point in Figure 23 is where the viscosity passes the $3000 \mathrm{cP}$ measurement. This is between the $10 \%$ and $20 \%$ particle loading which is way lower than the optimal loading. A loading closer to $50 \%$ is desirable in order to produce a green body that can be sintered to full densification. This means that for the Ceralox 
alumina and resin mixture, a vat recoating system will not work because the viscosity gets very high with the higher loading percentages. In order to fix this, the sample will be printed with a tape casting method so the alumina particles can be loaded to a higher percentage. Another observation when mixing these different test samples was the amount of sedimentation at different levels. After mixing the compounds and letting them sit for a minute, there was a very thin layer of alumina at the bottom. Once the mixture got to about $30 \%$ particle loading there was no noticeable sedimentation. This is due to the fact that the viscosity increased enough to hold those particles in place. When the particle loading went above $40 \%$, the viscosity was too high to be measured. It was a very thick sticky peanut butter consistency and would hold its shape without flowing.

\subsubsection{Combining and Printing}

In order to test the cure depth of the next mixture, a particle loading of $35 \%$ was printed. The step depth between each exposure was $.2 \mathrm{~mm}$ and a tape casting method was used to apply the resin. Rather than dipping it into the bath, the resin mixture was squeegeed onto the plate. Each exposed layer adhered to the previous layer so the cure depth was sufficient with this resin. The lack of photo inhibitors helped in this way compared to the zirconia example that did not have a deep enough cure. The final part can be seen in Figure 24 and shows the cylinder shape with some jagged edges between exposures. The next step is to increase the particle loading for this mixture and to improve the layer adhesion overlap. This way each layer is exposed directly over the original so that the edges have more of a smooth pattern. The first 
print with this new mixture was a success and gave a baseline to move onto the next steps.

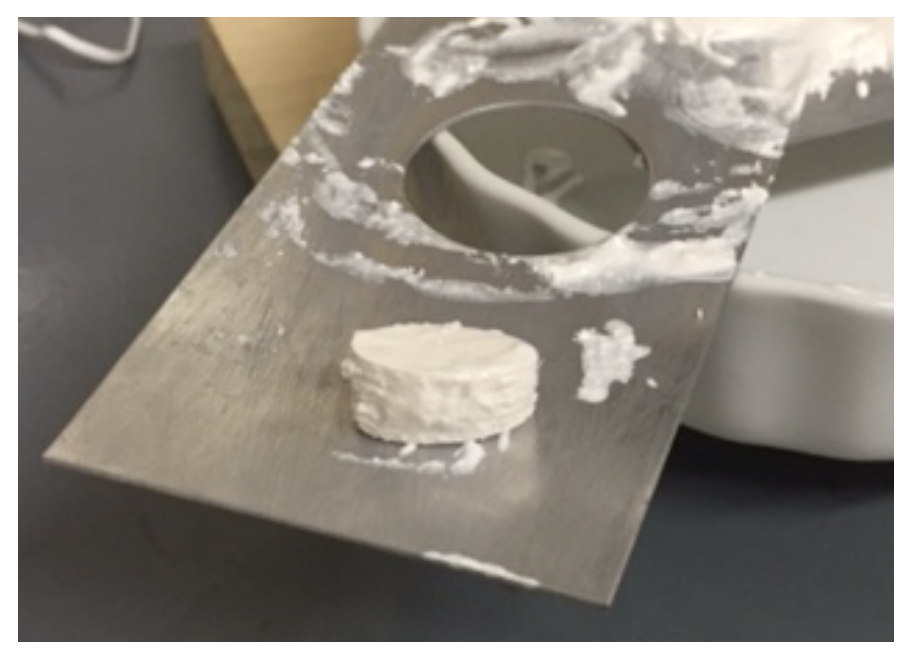

Figure 24. Ceralox alumina 35\% volume loading printed part

\subsection{Fifth Experiment (39\%)}

After confirming the compatibility of the Ceralox alumina and Spectra resin, the next step was printing a larger more complex part with different layer geometries. For this print, the resin was loaded to $35 \%$ alumina by volume initially. Instead of jumping up to $40 \%$ like the previous experiment, small amounts of alumina were added in $1 \mathrm{~g}$ increments to mix into the suspension. This helped to disperse the particles and keep the viscosity low instead of clumping up. The final mixture printed with was 39\% alumina because it was getting close to clumping up while mixing if any more was added. This part was also printed with a tape casting method due to the higher viscosity. Figure 25 shows the final printed part using the Cal Poly logo. Each layer has very high accuracy with regards to the features. The small disconnect between the $\mathrm{C}$ and $\mathrm{P}$ is kept separate throughout the print. The one discrepancy visible is the change between each height. As the platform lowers, the layer height gets thicker in 
some places and sometimes doesn't print directly on the previous layer. The thicker portions are due to the tape casting and not getting a perfectly smooth layer application. The disconnect between layers is due to the platform moving between layers while printing. In order to fix this, another bolt can be added to prevent any rotation on the printing plate. For the thicker portions, the ideal solution is getting the viscosity low enough to print in the vat instead of tape casting but this requires a new formulation. This was a successful print showing the capability of larger objects with complex features but there are still some modifications that can be made to improve.

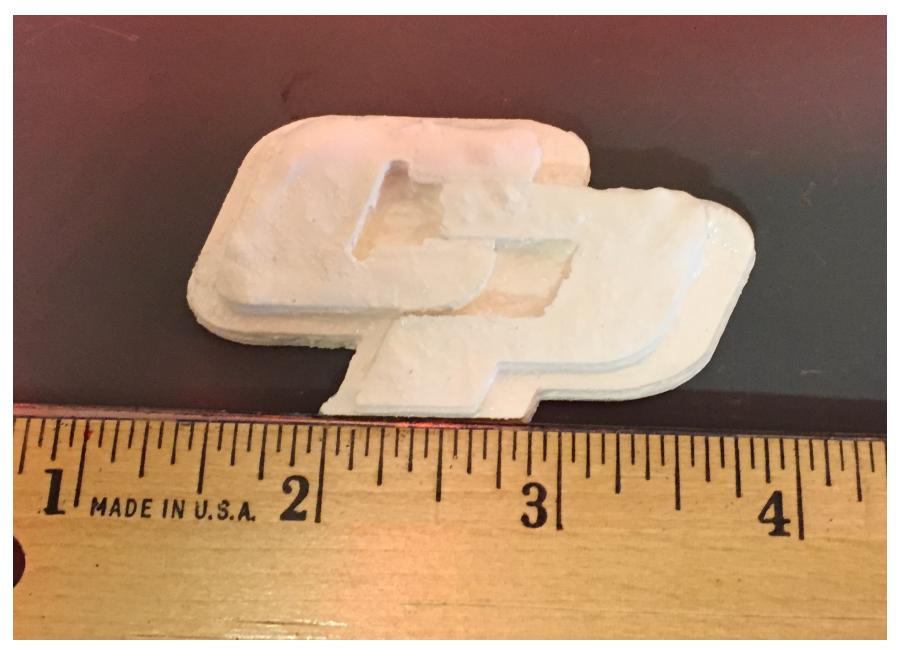

Figure 25. CP logo printed with $39 \%$ Ceralox alumina

\subsection{Sixth Experiment (40\%)}

For this print, the print plate had another bolt added to it to prevent any rotational movement that has caused misalignment in previous prints. Another change was the slow addition of powder to the resin. The Ceralox alumina was added in 1 gram increments after reaching $30 \%$ volume to slow the change in the viscosity. The gradual addition allowed for the resin to remain flowing rather than dumping all the powder in at once and struggling to mix completely. This slow addition allowed the mixture to get up to $40 \%$ volume loading without the viscosity getting too high so 
that it would not spread easily during tape casting. For this print, more layer detail was explored with the use of the Cal Poly Mustang logo. Three different sizes were used to see how the detail changed with part size. The results can be seen below in Figure 26.

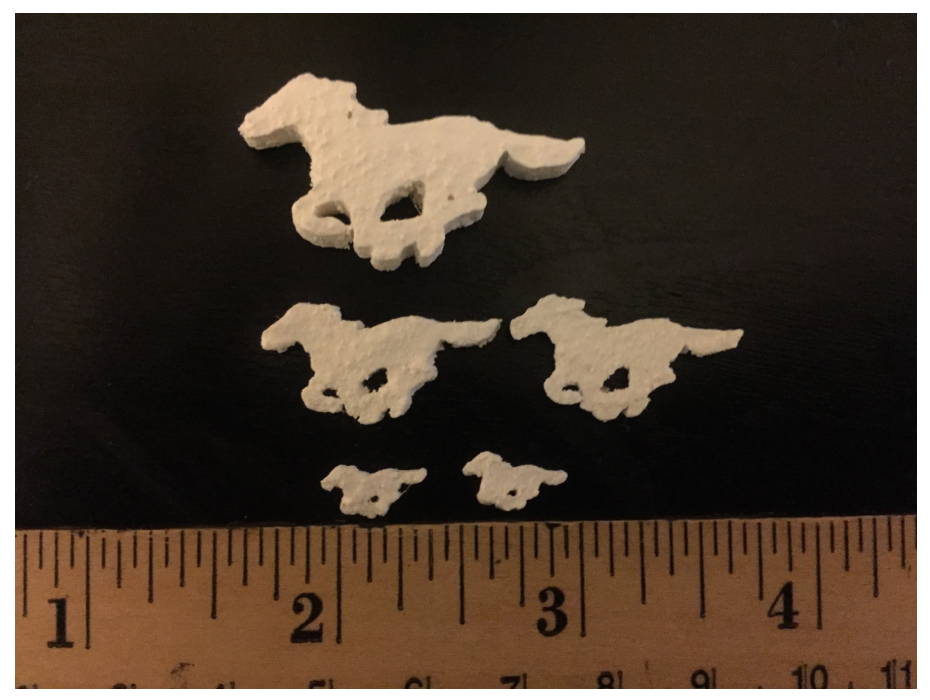

Figure 26. Cal Poly Mustang printed in three different sizes to compare details

Each subsequent layer had very good placement on the previous which confirms that the print plate no longer has rotational movement to take away from the accuracy. The parts are a quarter inch tall and the detail is held through each layer. The difficult part with these was the post processing. All of the mustangs had very good feature detail but it was hard to remove the resin from the cracks and holes. The smallest mustangs produced fine details but the post processing caused less accuracy. I can confirm this because I could feel with a pin tip the softer parts of the print but could not fully remove the excess resin. The gap between the front hoof is ten thousandths and it was held separate in all the prints which shows this printer has very good layer accuracy. Overall this was a successful print and it seems like a lot of the details are 
dialed in to provide high quality parts. The next part is taking these parts to the furnace to sinter them and see how they behave.

\subsection{Sintering}

With these green bodies manufactured from the printer, the next step was to create fully dense parts by putting them in a furnace. The furnace used for sintering these parts was a SunFire 10 shown in Figure 27. This has different ramp features and holds that are programmable in order to get the desired sintering process. It has a ceramic crucible that holds the part and raises up into the furnace where it is held at whatever temperature it is programmed to.

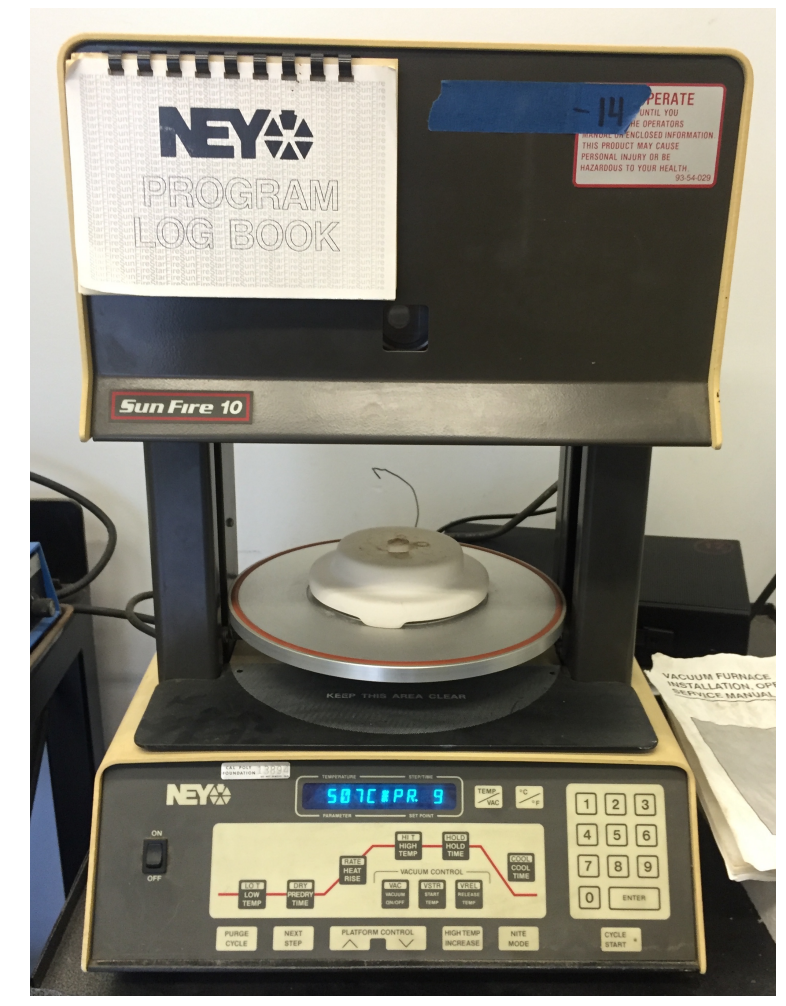

Figure 27. SunFire 10 furnace used to sinter alumina parts

The furnaces on campus all have a max range up to $1200^{\circ} \mathrm{C}$ which is typically too low for sintering alumina. Looking for other options, there was literature about low temperature sintering which we decided to attempt with the alumina samples. In one 
article, the researchers took a compressed high purity alumina and sintered it for two hours at $1275^{\circ} \mathrm{C}$ which was successful resulting in a $95.5 \%$ relative density part (Rao, Iwasa, \& Kondoh, 2000). Our test was to attempt this at $1200^{\circ} \mathrm{C}$ to see if we could get any sintering to occur with the cylindrical part. The first phase for our test was the resin burnout. For this, the furnace was ramped up at $25^{\circ} \mathrm{C} / \mathrm{min}$ to $732^{\circ} \mathrm{C}$ and held for 20 minutes. Figure 28 shows the part during resin burnout at approximately $400^{\circ} \mathrm{C}$. The black is the resin carbonizing and burning out of the part. After it reached $732^{\circ} \mathrm{C}$, the carbonizing was over and the part went back to white showing that the resin was gone.

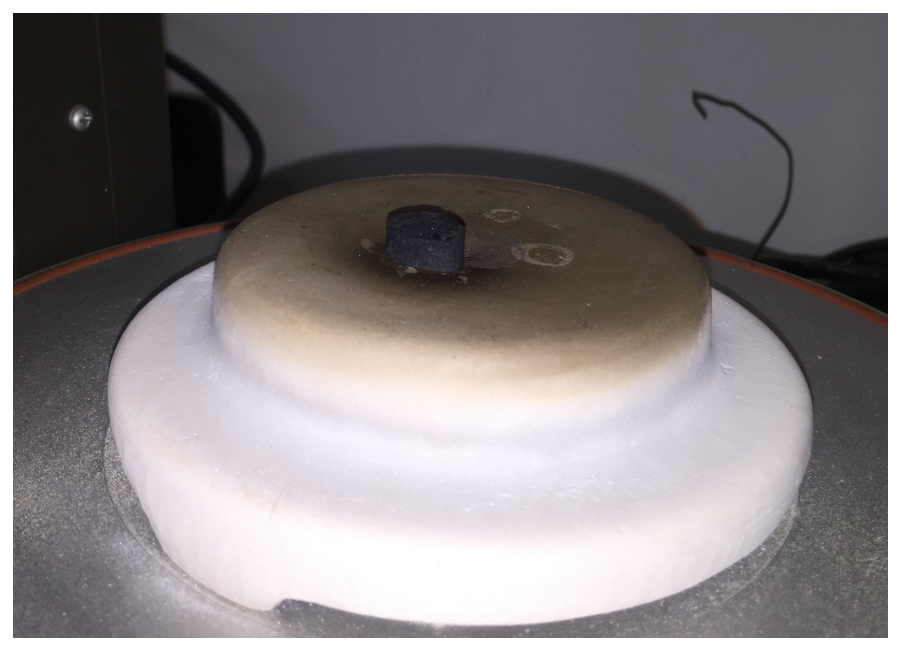

Figure 28. Resin burnout initial stages showing carbonization

The next phase was sintering which was done with a $.3^{\circ} \mathrm{C} / \mathrm{min}$ ramp up to the max of $1200^{\circ} \mathrm{C}$. This was held there for 5 hours because we were hoping that the time would help to alumina to grow together. At the end of the sintering, the part was red hot as seen in Figure 29. Figure 30 shows the part after it cooled down post sintering. 


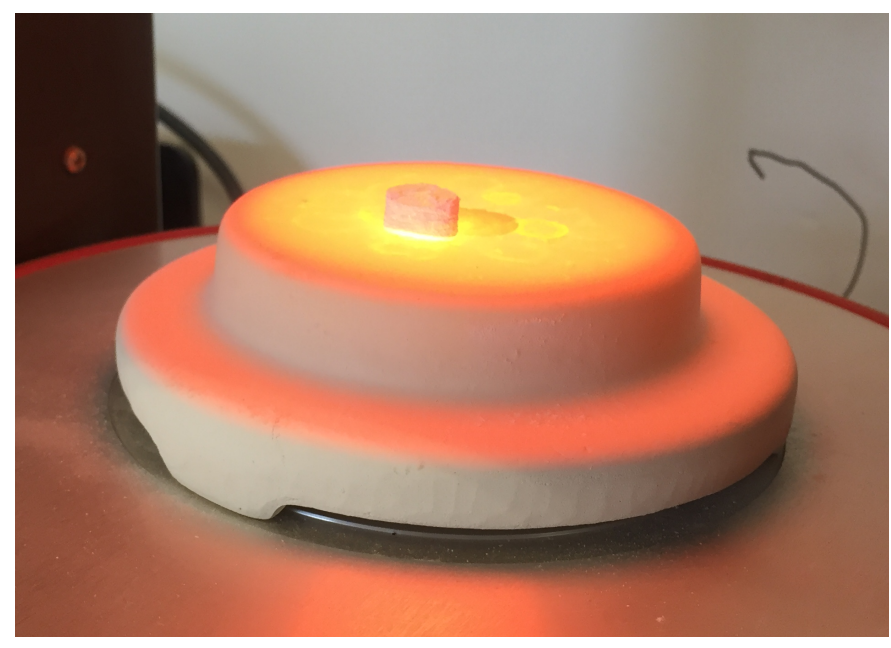

Figure 29. Post sintering of the part showing the red hot temperature

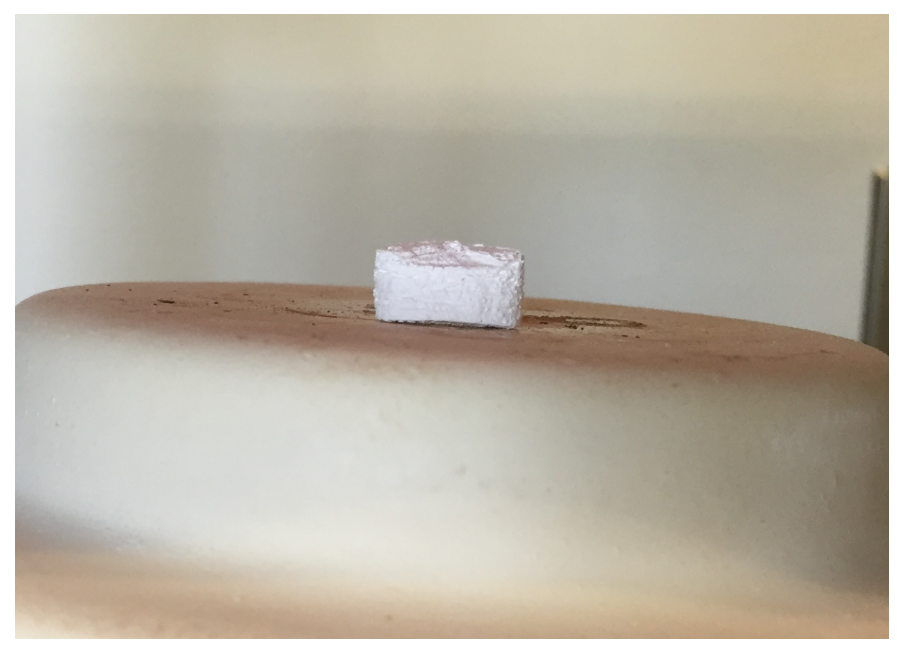

Figure 30. Cooled down part post sintering

The final result was an unsuccessful sintering. The part held its shape but it did not get dense enough to be considered successful. When the surface was scratched, it began to chip which indicated that the hardness was not where it should be. This low temperature sintering did not work in this application for two reasons. The first being that the temperature was not high enough as compared to the literature. Another factor is the fact that this part is not compressed. All the parts in literature had high compression values which may help with the sintering process at the lower 
temperatures. For our parts, we need a furnace that can reach $1600^{\circ} \mathrm{C}$ to see how it sinters. 


\section{COST COMPARISON}

Ceramic parts have optimal material properties that could be used for many consumer applications as well as research but far too often they are not implemented due to cost restrictions. The cost associated with designing, manufacturing, and producing ceramic parts eliminates the material as an option very early on. This mask projection stereolithography brings a cost effective machine alternative that can be refined to create production worthy parts. The most common current manufacturing of ceramics falls under replication, which includes die-casting, molding, and injection. This will be used to compare the costs with additive manufacturing. Replication manufacturing has five main costs associated with it; direct machine costs, indirect machine costs, machine operation costs, material cost, and tooling cost. Comparing these to the additive manufacturing process, most of these costs are similar. The indirect machine costs refer to floor space or storage of different parts which is similar for the two processes. Both will take up floor space in their respective applications. The machine operation costs is also similar which refers to the operator cost and power consumption. Looking at the average operator cost and power consumption costs, these account for a small part of the overall cost and are comparable. The material costs are disregarded because for this comparison, it is assumed that the same material being used. Compared to additive manufacturing, the main differences in cost fall under the direct machine cost and tooling costs. The next parts will look at the difference in cost based on machine cost and tooling cost for replication processes versus additive processes. 


\subsection{Ceramic Manufacturing Costs}

The first cost in ceramic manufacturing is having the machinery to press the dies. These machines hold the dies while the material is added or compress the powder into the mold so they need very high pressure. Typically the machines needed are specialized and very expensive (over $\$ 100,000$ ) so rather than purchasing the huge machine, it is contracted out to a vendor. This gets rid of the machinery cost but introduces other costs like paying the vendor and making the tooling. Once the part you want to have manufactured is designed, it needs to have tooling developed for it. This takes engineering time to design the die, which adds labor cost and time until production. Next the tooling has to be made which involves machining, grinding, and polishing to ensure part accuracy. The cost for just the die costs over $\$ 20,000$ dollars and since the parts are ceramic, they still have to be sintered in a furnace afterwards. There are many steps necessary to follow with this manufacturing process and in order to have a low part cost, you need to have a higher volume.

\subsection{Selective Laser Sintering}

Selective laser sintering uses a high power laser to fuse the ceramic particles together. For a machine with a print plate of about 4 inches $\mathrm{x} 4$ inches, it costs around $\$ 250,000$. In addition, the material is expensive as well because it needs to have a high purity and the correct particle size. Designing parts for this is much faster than ceramic manufacturing because once the $\mathrm{CAD}$ is made, it can be uploaded to the machine with only a few changes. Figure 31 shows a generic cost variation between manufacturing processes once the initial machines are purchased. Notice how the two additive manufacturing processes have a constant cost per unit while the die casting 
depends on the unit count. This is because of the tooling costs and the large affect they have. While selective laser sintering has a lower cost for smaller batch sizes, stereolithography has an even lower material and printing cost.

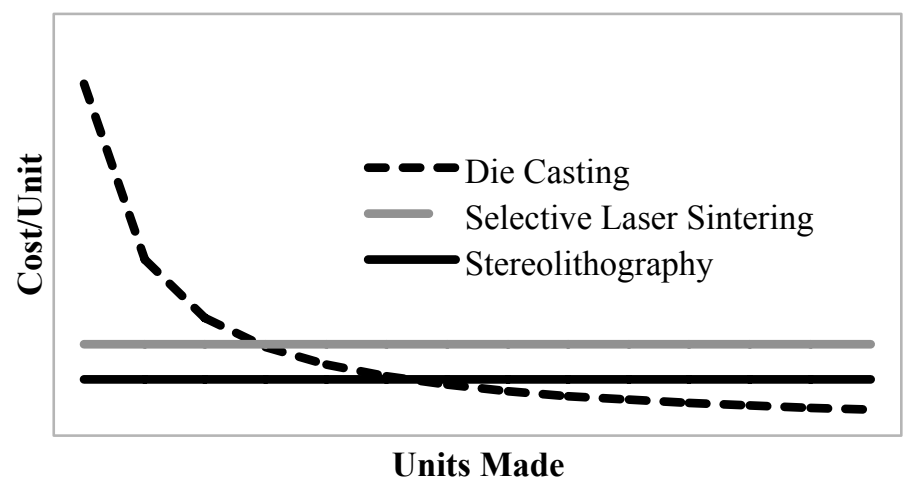

Figure 31. Cost comparison between different ceramic manufacturing processes

\subsection{Stereolithography}

Stereolithography additive manufacturing has a wide array of printers available to the public. The production printers with larger print plates start at around $\$ 20,000$ for the machine. These typically have faster printing rates as well. For a much lower price point, there are desktop printers with very similar resolution. Stereolithography remains the most accurate form of 3D printing and the difference between desktop and industrial are a few microns. The other difference is the light source used. Industrial printers use a vector scan approach with lasers while desktop printers use a mask projection. These desktop printers start at $\$ 3,500$ for the lowest model. One difference with these printers is that they use mask projection from the base. This means that the lightsource prints through a glass plate and exposes on the bottom side of a print plate. Then the part is slowly pulled out of the resin instead of building into it. Currently, these printers are only able to print resin parts. For ceramic 
stereolithography, the viscosity and density of the material is too high to print with on the commercial printers. The viscosity is typically too high to flow under this plate between prints and if it were able to print, the ceramic materials have a significant weight. This would cause the part to pull off from the print plate. A solution to this is the prototyped top mask projection ceramic stereolithography printer from this thesis.

\subsection{Top Mask Projection Ceramic Stereolithography}

The prototype developed in this thesis is able to print both polymer parts and ceramic parts. It is the first top mask projection printer used for ceramics. Previous researchers have used vector scanning methods for printing but these have a much higher cost. Table 4 shows the cost breakdown for the materials required to make the printer. As you can see it is much cheaper than all other current ceramic manufacturing processes for the startup machine cost. The main percentage of cost comes from the lightsource, which provides the high resolution.

Table 4. Cost breakdown of ceramic stereolithography prototype printer

\begin{tabular}{|l|r|c|}
\hline \multicolumn{1}{|c|}{ Component } & Cost (\$) & \% of Total \\
\hline Wintech Pro4500 Lightcrafter & 1,995 & 76.8 \\
\hline Thorlab Single Axis Translation & 272 & 10.5 \\
\hline Thorlab Right Angle Bracket & 73 & 2.8 \\
\hline Structural Support w/ Fasteners & 20 & 0.8 \\
\hline Print Plate & 7 & 0.3 \\
\hline Ceramic Vat & 5 & 0.2 \\
\hline 500 grams Ceralox alumina & 100 & 3.9 \\
\hline 500 mL Spectra Resin & 125 & 4.8 \\
\hline Total & $\mathbf{2 , 5 9 7}$ & $\mathbf{( 1 0 0 )}$ \\
\hline
\end{tabular}

As far as material costs go, the main cost increase comes from the price of the ceramic. Since commercial resins are being used, the price per $\mathrm{mL}$ for resin is the 
same for both current stereolithography and with ceramic. The cost increases because a lot more ceramic has to be added to bring the volumes equal for both processes. From a volume perspective, the resin only printer would cost $\$ 125$ for $500 \mathrm{~mL}$ of the current material tested. The ceramic stereolithography at $40 \%$ volume loading would cost $\$ 75$ for $300 \mathrm{~mL}$ of resin and $\$ 158$ for $790 \mathrm{~g}$ of alumina to total $\$ 233$ for $500 \mathrm{~mL}$ of printing material. This shows an $86 \%$ increase in the cost for materials. On a large scale, this is a large cost difference but typically a part will only use $20 \mathrm{~mL}$ of material. This translates to a $\$ 5$ resin part and $\$ 9.32$ ceramic part, which is a very similar part cost for smaller scale production.

Labor for current resin stereolithography consists of taking the CAD, converting it to slices for the printer, and then post processing the parts. For this ceramic stereolithography, additional labor is needed to mix the ceramic and resin, manually apply each layer, and then post process and send to a furnace for sintering. The longest of these steps is the manual layer application during printing which takes however long the print time is. In future revisions, this step will be eliminated and made automatic but since this is a prototype, most of the labor cost is focused here. For cost analysis purposes, we will look towards a more commercially ready ceramic stereolithography printer with automated features. The resin only stereolithography totals approximately one hour assuming the software is efficient and no extensive post processing is needed. For ceramic stereolithography of the same part, an additional 2 hours would be needed. The mixing is a slow process where the ceramic is slowly added to keep the viscosity low. The post processing takes longer as well because the viscosity is higher and the free ceramic particles tend to stick to the part. 
Ceramic stereolithography would take three times as long as pure resin so that is three times more cost. With a $30 \$$ hour work rate, this translates to $\$ 60$ additional cost for ceramic.

Looking at these different cost parameters, the ceramic stereolithography has a lower initial machine cost but the labor and material have a higher cost. As more parts are created, the cost of the ceramic stereolithography will pass the resin printer cost due to recurring operation. However, the quality of the parts is important here because the higher cost will give you a more structural part with better material properties than polymers. The ceramic parts may begin to cost more but it is a much better alternative to current ceramic manufacturing processes. With this printer, it is the first step to showing the possibility of a lower cost alternative to producing ceramic parts and will make it more accessible to the public. 


\section{CONCLUSIONS AND RECOMMENDATIONS}

The objective of this research was to test the feasibility of using commercially available resins to produce ceramic green-bodied parts with a top mask projection stereolithography printer at a low cost. To explore this, a desktop stereolithography

printer was designed and built to generate these parts as described in this thesis. Then a series of resin and ceramic powders were tested in conjunction with each other to explore the print quality and viscosity. The best print results came with the Ceralox alumina and Spectra resin because it gave the largest cure depth out of any other mixtures. The viscosity was very high for all of the mixtures with high ceramic loading which resulted in the parts having to be printed with a tape casting method. When the parts were put into the furnace, the binder was all burned out but the ceramics did not reach full density due to the lower sintering temperatures used. In the end, this printer was able to produce ceramic green bodies at a low cost compared to current ceramic manufacturing techniques, opening it up as an alternative process for certain engineering applications. Comparing this ceramic stereolithography printer with previous research performed in this field, this process has a few unique aspects. First, this printer uses a mask projection technology in order to expose the resin with UV light. All other researchers have used a vector approach which involves a HeCd laser or UV laser to cure the resin point by point. These machines are much more expensive to produce and have a slower printing process. In addition, the resins and ceramic powders tested were available commercially and mixed by distributors. This allows more accessible materials to be used with this printer and less chemical knowledge necessary to 
develop a suitable resin. This combination provides new research on the ceramic stereolithography front for low cost manufacturing.

As far as printing tests, more research should be done as to the extent of what features can be produced. This includes ramped faces on the part which relies heavily on cure depth for resolution. Also overhanging geometries will determine if the cure depth is a problem and causes some overcuring. Another change to the print is starting with a generic sacrificial base on the print plate. This should allow for easier removal of the parts with no features being broken on the print plate.

This initial prototype has given a good foundation to move forward with and there are some improvements that can be made to the physical printer. One upgrade is making the printer fully automatic. This would involve changing the $\mathrm{z}$ stage to an automatic ball screw controlled by a board and a software that can sink this with the exposure timing. This should only be done if the viscosity is able to be brought below $3000 \mathrm{cP}$ so that the vat could accomplish the recoating. If the viscosity remains high, an automatic printer would not change very much because it would still need to be tape cast. Another improvement could be making the structural supports out of aluminum. The poplar base works well for the short term but as the printer gets older, the structural stability may begin to change and potentially fall apart. In addition to the mechanical improvements, the rheological properties should be explored in order to bring the viscosity lower. One recommendation is using dispersants to initially wet the ceramic and then mix it with the resin. This can help bring the end viscosity down because of the lower agglomeration present. One challenge with this is finding a commercial supplier with a dispersant that is 
compatible with the resin material. Another test is finding ceramics with smaller particle sizes because the smaller the particle size, the lower the viscosity. As the particle sizes decrease, the cost increases exponentially so it would be optimal to find the point where cost outweighs the benefit. Another change is the mixing techniques used to mix the ceramic and resin. A lot of researchers use ball milling to make sure the ceramic is dispersed so if this technology is available, it can help to lower viscosity. Finally a new resin can be explored with different properties. The resins used in this research are acrylic based while there are some epoxy based resins on the market. Epoxies typically have a much lower viscosity but require more power and time to cure the resin. This may bring the viscosity low enough to recoat using the vat but the following question would be if the lightsource is powerful enough.

One recommendation to explore that was briefly covered in this paper is the sintering of the green-bodies. A high temperature furnace that can go up to $1600^{\circ} \mathrm{C}$ is required for this. Then the final density of the green parts can be measured to see how the loading volume affects the final part. It will also show the shrinkage that occurs which can be translated into how much to oversize the initial printed part. Overall, this thesis helps to explore the feasibility of mask projection in ceramic stereolithography and take the first steps towards a lower cost alternative. The prototype gives a starting point to see all the factors that affect the printing process and which variables have the largest affect on the outcome. Future research will go towards improving the current prototype and making this printing process more reliable and accessible for ceramic manufacturing applications. 


\section{REFERENCES}

August, C. R. (2010). Examination of particle/particle interactions and their impact on rheology and mixedness of an alumina/titania system. (PhD Thesis), Retrieved from http://dx.doi.org/doi:10.7282/T3125SVQ.

Bonnoit, C., Darnige, T., Clement, E., \& Lindner, A. (2010). Inclined plane rheometry of a dense granular suspension. Paris University. Paris: Laboratoire de Physique et Mécanique des Milieux Hétégogènes.

Brady, G. A., \& Halloran, J. W. (1997). Stereolithography of ceramic suspensions. Rapid Prototyping Journal , 3 (2), 61-65.

Brookfield. More Solutions to Sticky Problems. Middleboro, MA: Brookfield Engineering Labs.

Eschl, J., Blumenstock, T., \& Eyerer, P. (1999). Comparison of the Curing Process of Epoxy and Acrylate Resins for Stereolithography by Means of Experimetal Investigations and FEM-Simulation. Solid Freeform Fabrication Symposium , 453-460.

Gibson, I., \& Rosen, D. (2010). Additive Manufacturing Technologies Rapid Prototyping to Direct Digital Manufacturing. Boston, MA: Springer Science Business Media, LLC.

Griffith, M., \& Halloran, J. (1996). Freeform Fabrication of Ceramics via Stereolithography. American Ceramic Society .

Hatzenbichler, M. (2013). Additive Manufacturing of Photopolymers using the Texas Instruments DLP Lightcrafter. Emerging Digital Micromirror Device Based Systems and Applications , 8618.

Hatzenbichler, M. G. (2012). DLP Based Light Engines for Additive Manufacturing of Ceramic Parts. SPIE, 8254.

Hinczewski, C., Corbel, S., \& Chartier, T. (1998). Ceramic Suspensions Suitable for Stereolithography. European Ceramic Society, 18, 583-590.

Hopkinson, N., \& Dickens, P. (2001). Rapid Prototyping for Direct Manufacture. Rapid Prototyping Journal , 197-202.

Jacobs, P. (1996). Stereolithography and other RP\&M technologies. Society of Manufacturing Engineers .

Jefri, M., \& Zahed, A. (1989). Elastic and Viscous Effects on Particle Migration in PlanePoiseuille Flow. Journal of Rheology . 
Kirihara, S. (2009). Structural Joining of Ceramics Nanoparticles. KONA Powder and Particle Journal , 27.

Liao, H. (1997). Stereolithography Using Compositions Containing Ceramic Powders. (PhD Thesis), Interlibrary Loan \#291679.

Rao, P., Iwasa, M., \& Kondoh, I. (2000). Properties of low-temperature-sintered high purity $\alpha$-alumina ceramics. Journal of Materials Science Letters , 19 (7), 543-545.

Riedel, R., \& I-Wei, C. (2008). Ceramics Science and Technology (Vol. 3). Weinhelm.

Ritzhaupt-Kleissl, H. (2009). Ceramics Processing in Microtechnology. Scotland, UK: Whittles Publishing.

Thomas, D. S. (2014). Costs and Cost Effectiveness of Additive Manufacturing. NIST Special Publication 1176 .

Viscosity Values Chart. (n.d.). Retrieved January 12, 2016, from InnoCal Solutions: http://innocalsolutions.com/tiarticles/tiairandfluidquantities/56-viscosity-values

Wang, H., Zhou, W., \& Li, D. (2010). A novel aqueous ceramic suspension for ceramic stereolithography. Rapid Prototyping Journal , 16 (1), 29-35.

White, G. (2013). Economic Analysis of Additive Manufacturing for Final Products: An Industral Approach. University of Pittsburgh Swanson School of Engineering .

Xu, G., Luo, S., Ma, X., \& Yang, J. Influences of Building Parameters on Over-cured Depth in Stereolithography System. 2010 International Conference on Measuring Technology and Mechatronics Automation.

Zhiwei, G., Jianhua, M., \& Shuhuai, H. (2006). Development of a Hyprid Photopolymer for Stereolithography. Journal of Wuhan University of Technology, 21 (1). 